\title{
Urban Form, Growth, and Accessibility in Space and Time: Anatomy of Land Use at the Parcel-Level in a Small to Medium-Sized American City
}

\author{
Long Zhou ${ }^{1, *}$, Guoqiang Shen ${ }^{1,2}$, Yao Wu ${ }^{1}$, Robert Brown ${ }^{1,3}$, Tian Chen ${ }^{4}$ and Chenyu Wang ${ }^{1}$ \\ 1 Faculty of Urban Management and Studies, City University of Macau, Macau 999078, China; \\ guoqiang.shen@uta.edu (G.S.); ywu@cityu.mo (Y.W.); R.Brown-4@plymouth.ac.uk (R.B.); \\ U17091105085@cityu.mo (C.W.) \\ 2 College of Architecture, Planning and Public Affairs, University of Texas at Arlington, \\ Arlington, TX 76019, USA \\ 3 School of Arts and Humanities, Plymouth University, Plymouth PL4 8AA, UK \\ 4 College of Architecture, Tianjin University, Tianjin 300072, China; chentian5561@vip.sina.com \\ * Correspondence: lzhou@cityu.mo; Tel.: +86-853-85902862
}

Received: 8 November 2018; Accepted: 30 November 2018; Published: 3 December 2018

\begin{abstract}
Using the City of Corvallis, Oregon, a small to medium sized American city, as a test-bed, this paper examines the City's urban growth in relation to urban accessibility. This relationship is explored in an anatomic spatial-temporal fashion, taking account of: the number and size of developed land use parcels over time; urban accessibility from residential to non-residential land use areas; and the statistical relationships between urban form and urban accessibility. This investigation of land use is structured around use-classification and examined within a range of dimensional and demographic measurements over 5-year time periods from 1853 to 2014; concurrently, urban accessibility is measured by the least-cost path distance as calculated through the OD cost matrix analysis in GIS. The results indicate that the city grew spatially at different rates and its urban accessibility experienced both ups and downs over time. The city's population growth corresponded closely with urban growth and its decreasing population density negatively impacted on the city's urban accessibility to commerce, industry, and office for most time periods. Significantly, while the urban density increased steadily after 1950s concurrent with an increase in urban sprawl, in contrast to previous studies on the metropolitan condition, the urban density had no evident impact on urban accessibility in Corvallis. Instead, increasing the land-use mix was a more effective and feasible approach to reduce urban travel path distance and enhance accessibility than increasing population density or urban development density. Accordingly, this research provides evidence-based policy recommendations for planning sustainable urban mobility and urban form in small to medium-sized cities.
\end{abstract}

Keywords: urban form; urban growth; accessibility; land use and parcel; space and time; scatterplot matrix and correlation

\section{Introduction}

Urbanization has been altering human settlements drastically since the beginning of the 20th century [1]. Urban transportation and land use are two important components of urbanization and play major roles in urban physical expansion, economic development, and social lifestyle change [2,3]. By 2011 , about $3 \%$ of the earth's surface had been urbanized, with $50 \%$ of the world's population living in cities, and 10 billion urban trips per day made worldwide [4]. It is predicted that the urban travel distances (UTD) in 2020 will be three times of that in 2000 as urban growth continues [5]. 
The substantial increase of travel distances in cities is largely attributed to sprawling urban residential land uses, hence accessibility to non-residential land uses is decreasing [6]. Studies have also indicated that urban form, characterized by urban size, density, and land-use mix, influences accessibility to urban facilities significantly, especially in megacities [7-9].

However, the spatial-temporal dynamic nature of urban growth, urban form, urban accessibility, and their relationships is worthy of further studies [10], especially for small to medium-sized cities as self-standing municipalities outside major urban agglomerations and playing an important role in urban and regional systems [11-14]. Using the City of Corvallis, Oregon, ranked as the third best small to medium-sized city to live in U.S. [2] as the test-bed, this study analyzes Corvallis's urban form (urban density and diversity), growth (population, size and real estate's value), and accessibility (measured by least-cost path travel distance), and identifies their patterns and relationships. The objective of this research is to answer the following two questions on the spatiality of Corvallis with respect to the City's current boundary and in time through the City's history between 1853 and 2014: (1) What are the City's growth patterns and accessibility changes by land use at the parcel level? (2) How are urban growth, urban accessibility, and urban form statistically related?

Urbanization is characterized by population growth, economic growth [15], suburban development [16,17], and social differentiation [18]. Associated with the process of urbanization, urban growth, form and accessibility to non-residential urban facilities have posed planning and development challenges for many cities [19]. Urban growth refers to the social-demographic growth, such as population and economic development. Urban form refers to the overall spatial representation of various land use distributions across space and over time. It can be regarded as an aggregate spatial response to human activities or needs in a city [20]. For example, residential activities call for residential homes and neighborhoods, which together constitute residential land use. Similarly, non-residential activities, such as manufacturing, shopping, and business activities require industrial, commercial, and office land uses. Urban form is often described by urban size (urbanized area), urban density, and land-uses mix, which is regarded as relevant to urban trips, travel distance or accessibility to some degree [21]. Urban density is typically represented by a certain measure per unit space [22], such as population (population density [23]), building square footage (floor area ratio [24]), or housing unit (urban development density [25]), People living in high urban density areas enjoy significantly shorter commuting distances than those living in low urban density areas $[19,26]$. High urban residential density neighborhoods are mostly located closer to jobs, shops, parks, schools and other urban facilities or amenities [27]. Urban land-use mix, often referred to as the degree of residential and non-residential land use combination, is measured as the number or share of diverse land-uses per unit of land area [19]. Land-use mix is considered as an essential principle in sustainable growth policies [28]. People living in higher land-use mix districts have shorter travel distances, higher accessibility from residential land use to non-residential land use and more sustainable travel behaviors [29,30]. Previous studies and practices indicate that good urban form with proper density, balanced accessibility for shorter commuting, and optimized mix of land uses are crucial for good urban growth [31,32].

Urban accessibility is a fundamental spatial connection measure of urban activities for various parts of a city [33]. It can be used to examine spatial distributions of urban land uses, and hence urban form [9]. Trips made by people between urban land uses are often classified into various types, such as home-based trips between residential and non-residential land uses. For example, commuting trips are home-based work trips by people traveling between homes and their work places. The commuting trip is one major and perhaps the most important urban travel type, which helps explain jobs-housing balance, urban spatial structure, and spatial mis-match issues [34]. Urban trips and urban accessibilities are often and simplest measured by distance or time [35-37]. In general, the larger a city is, the longer the average trip traveling across the land use parcels in the city, and hence, the smaller the urban accessibility [38].

The above features of the urban built environment and their mutual influences and impacts have been studied quantitatively [39-44]. However most of them focus on megacities and very little research 
explores how the urban form develops and accessibility changes through a city's history and how the urban form features are related to accessibility for the small to medium-sized cities.

With the detailed parcel data on the developed land of Corvallis, a typical small to medium-sized city in the United States, this paper studies the City's urban form, growth, accessibility in time (from its birth to its present day) and in space (with respect to its current urban growth boundary). This is advanced by investigating urban growth and urban form at the land-use and parcel levels, calculating accessibilities from residence to other land-uses for all historical periods, and establishing strong statistical relationships for these built urban features; these parameters are the focus here as each contributes to the improvement of the urban accessibility and the sustainable planning and design of the urban spatial-temporal structures in the small to medium-sized cities.

\section{Material and Methodology}

Using the City of Corvallis, Oregon, as a test-bed, this study provides an exploratory and anatomic analysis of the City's urban form, growth and accessibility from residential to nonresidential land use parcels through the spatial analysis and origin-destination (OD) cost matrix analysis in GIS. Then this study identifies the relationships between the urban form variables with urban accessibility through the Pearson correlation model.

\subsection{Study Area}

The City of Corvallis is the county seat of Benton County, State of Oregon, U.S. It is located near the middle of the Willamette Valley. The City is within 90 mins drive of the Portland Metropolitan area, and it is located in a basin of 42 square miles [45]. It is ranked as the third best small to medium suburban city to live in the U.S. in 2018 by Livability [2]. The State and City's planning system is considered to be very successful in the U.S. by policy-makers and planners in terms of directing urban growth into urban growth boundaries and conserving more ecological values $[40,46]$. The first parcel in the City was established in 1853, and by 2014, there had been nearly fourteen thousand parcels supporting 55,298 people [47]. According to the Corvallis Comprehensive Plan, the area circumscribed by the City's current Urban Growth Boundary (UGB) is 28 square miles [48], which is the focus of this study. $35 \%$ of the land within the UGB had been urbanized by 2014 , within which parcels for residential, commercial, industrial, office, and open space land occupy $77 \%, 1 \%, 7 \%, 3 \%$ and $12 \%$, respectively (see Figure 1) [49]. The first modern transportation lines in Corvallis was constructed in 1889; and about 73 percent of workers commuted by the private automobile in 2013 [50].

The Planning Division in the City of Corvallis provided an open-access tax-lot parcel database. It documents each parcel within the UGB, including attributes such as the year built (urbanization year), land use category, building finished size (square footage), parcel size (land acreage), land value, improved value [49]. Using the year built attribute, this study sliced the City urban land use spatial distribution and growth history from 1853 to 2014 into 5-year intervals and grouped the land parcels accordingly. Given that the City's first land use classification started in 1897, we further grouped the land parcels by six major land uses (residence, commerce, industry, office, open space, and undeveloped). 


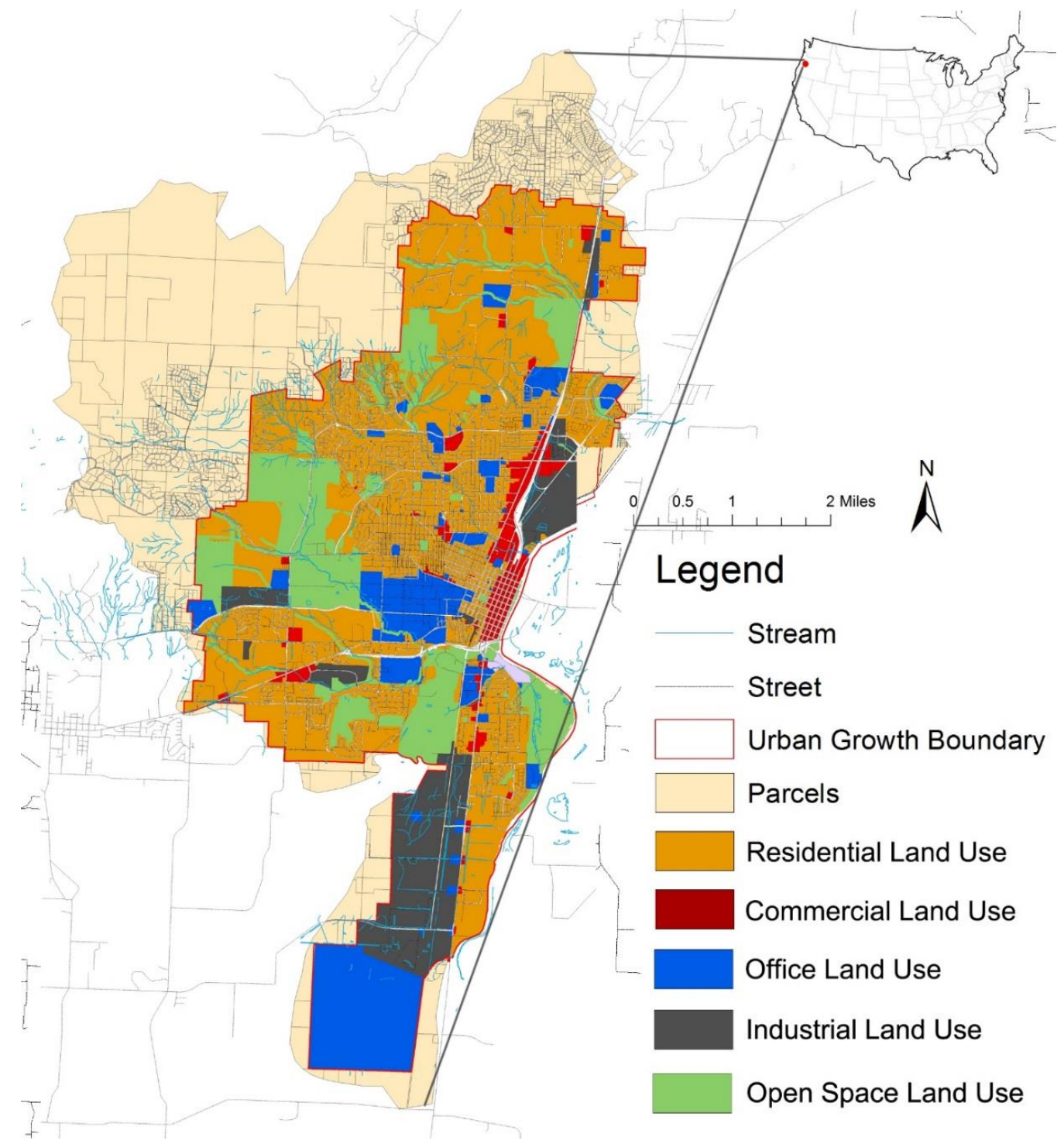

Figure 1. Urban Growth Boundary and Land Parcels and Land Uses in Corvallis.

\subsection{Urban Form and Growth Model}

Let $r \in R, c \in C, i \in I, f \in F, o \in O, u \in U$ denote indices for residential, commercial, industrial, office, open space, undeveloped parcels, respectively. Let $t \in T$ denote time intervals for Corvallis's urban history from 1853-2014. For each time period $t$, denote $P O^{t}$ as the City's population (population data before 1883 are not available), $P^{t}$ as the number of urbanized parcels; denote $A_{r}^{t}$, as the residential to non-residential parcel accessibility measures (least-cost path distance); $B S^{t}$ as the building square footage; $P D^{t}$ as population density; $U D^{t}$ as building square footage based density (floor area ratio) and $D U^{t}$ as urbanization development density. Denote $T S^{t}$ as the total city size in acreage; $U S^{t}, S^{t}, R S^{t}, C S^{t}, I S^{t}, F S^{t}, O S^{t}$, or $A S^{t}$ as undeveloped, total urbanized, urbanized residential, commercial, industrial, office, open space, or average urbanized parcel size in acreage, respectively. Similarly, denote $T P^{t}$ as the total parcels numbers; $U P^{t}, P^{t}, R P^{t}, C P^{t}, I P^{t}, F P^{t}$, or $O P^{t}$ as the total undeveloped, urbanized, residential, commercial, industrial, office, or open space parcels numbers, respectively. Let CM, IM, FM, OM, LM denote the residential-commercial parcels mix degree, residential-industrial parcels mix degree, residential-office parcels mix degree, residential-open space parcels mix degree and residential-nonresidential parcels mix degree, respectively. For each parcel, denote $L V^{t}, I V^{t}, A V^{t}$, and $M V^{t}$ as its land, improved, assessed, and market value, respectively. The abbreviations for variables are listed in Appendix A. 
With the notation above, we can derive the following relationships for urban form and growth measures as: $T S^{t}=U S^{t}+S^{t} ; T P^{t}=U P^{t}+P^{t} ; R S=\sum_{t} R S^{t}, R S^{t}=\sum_{r} R S_{r}^{t} ; C S=\sum_{t} C S^{t}, C S^{t}=$ $\sum_{c} C S_{c}^{t} ; O S=\sum_{t} O S^{t}, O S^{t}=\sum_{o} O S_{o}^{t} ; I S=\sum_{t} I S^{t}, I S^{t}=\sum_{i} I S_{i}^{t}$. Also, we can derive urban land parcel numbers as $R P=\sum_{t} R P^{t}, R P^{t}=\sum_{r} R P_{r}^{t} ; C P=\sum_{t} C P^{t}, C P^{t}=\sum_{c} C P_{c}^{t} ; F P=\sum_{t} F P^{t}, F P^{t}=\sum_{f} F P_{f}^{t}$; $O P=\sum_{t} O P^{t}, O P^{t}=\sum_{o} O P_{o}^{t} ; I P=\sum_{t} I P^{t}, I P^{t}=\sum_{i} I P_{i}^{t}$. In a similar fashion, we can define urban accessibility measures for different land uses as $C A_{r}^{t}=\sum_{c} C A_{r c}^{t}, C A^{t}=\sum_{r} C A_{r}^{t} ; F A_{r}^{t}=\sum_{f} F A_{r f}^{t}, F A^{t}=$ $\sum_{r} F A_{r}^{t} ; I A_{r}^{t}=\sum_{i} I A_{r i}^{t}, I A^{t}=\sum_{r} I A_{r}^{t} ; O A_{r}^{t}=\sum_{o} O A_{r o}^{t}, O A^{t}=\sum_{r} O A_{r}^{t}$. Finally, we can define land use mix measure $L M^{t}$, which includes four parts: commercial mix $C M^{t}=\left(C S^{t} * C P^{t}\right) /\left(S^{t} * P^{t}\right)$, office mix $F M^{t}=\left(F S^{t} * F P^{t}\right) /\left(S^{t} * P^{t}\right)$, industrial mix $I M^{t}=\left(I S^{t} * I P^{t}\right) /\left(S^{t} * P^{t}\right)$, and open space mix $O M^{t}=\left(O S^{t} * O P^{t}\right) /\left(S^{t} * P^{t}\right)$.

Based on the concept and measurement of urban form [22-25,36], the urbanized area or size, population density, urban density, and the land-use mix are calculated in Equations (1)-(5) respectively.

$$
\begin{gathered}
S^{t}=R S^{t}+C S^{t}+I S^{t}+F S^{t}+O S^{t} \\
P D^{t}=P O^{t} / S^{t} \\
U D^{t}=B S^{t} / S^{t} \\
D U^{t}=B S^{t} / P^{t} \\
L M^{t}=C M^{t}+I M^{t}+F M^{t}+O M^{t}
\end{gathered}
$$

\subsection{Urban Accessibility Model}

The urban accessibility is measured by the average of the least-cost path distances [36,37] between origins and destinations (OD). OD distance matrixes were built in GIS via direct distances between centroids of residential land use parcels to all non-residence land use parcels [51]. The total of the average travel distances from residence to non-residence was used to indicate overall accessibility in the City.

\subsection{Correlation and Regression Models for Urban Form and Accessibility}

To identify the relationships of urban form characteristics with urban accessibility from residence to non-residential land-uses for Corvallis through the urban growth history, we constructed the Pearson correlation model as follows:

$$
\begin{gathered}
\left(T A^{t}, P O^{t}, B S^{t}, P^{t}, S^{t}, P D^{t}, U D^{t}, L M^{t}, M V^{t}\right) \\
\text { vs. }\left(T A^{t}, P O^{t}, B S^{t}, P^{t}, S^{t}, P D^{t}, U D^{t}, L M^{t}, M V^{t}\right)
\end{gathered}
$$

\section{Results and Discussions}

\subsection{Urban Growth Patterns and Accessibility Changes}

Figure 2 shows the urban form and growth for the six land-uses by 5-year period from 1893 to 2014. The data from 1853 to 1892 were omitted since office parcels only started in 1893 . Clearly, the total urbanized parcels generally grew, though not strictly increasing, from 18 parcels by 1, 897 to 373 by 1942, to 1189 by 1992, and with a peak of 1323 parcels by 1997 . 


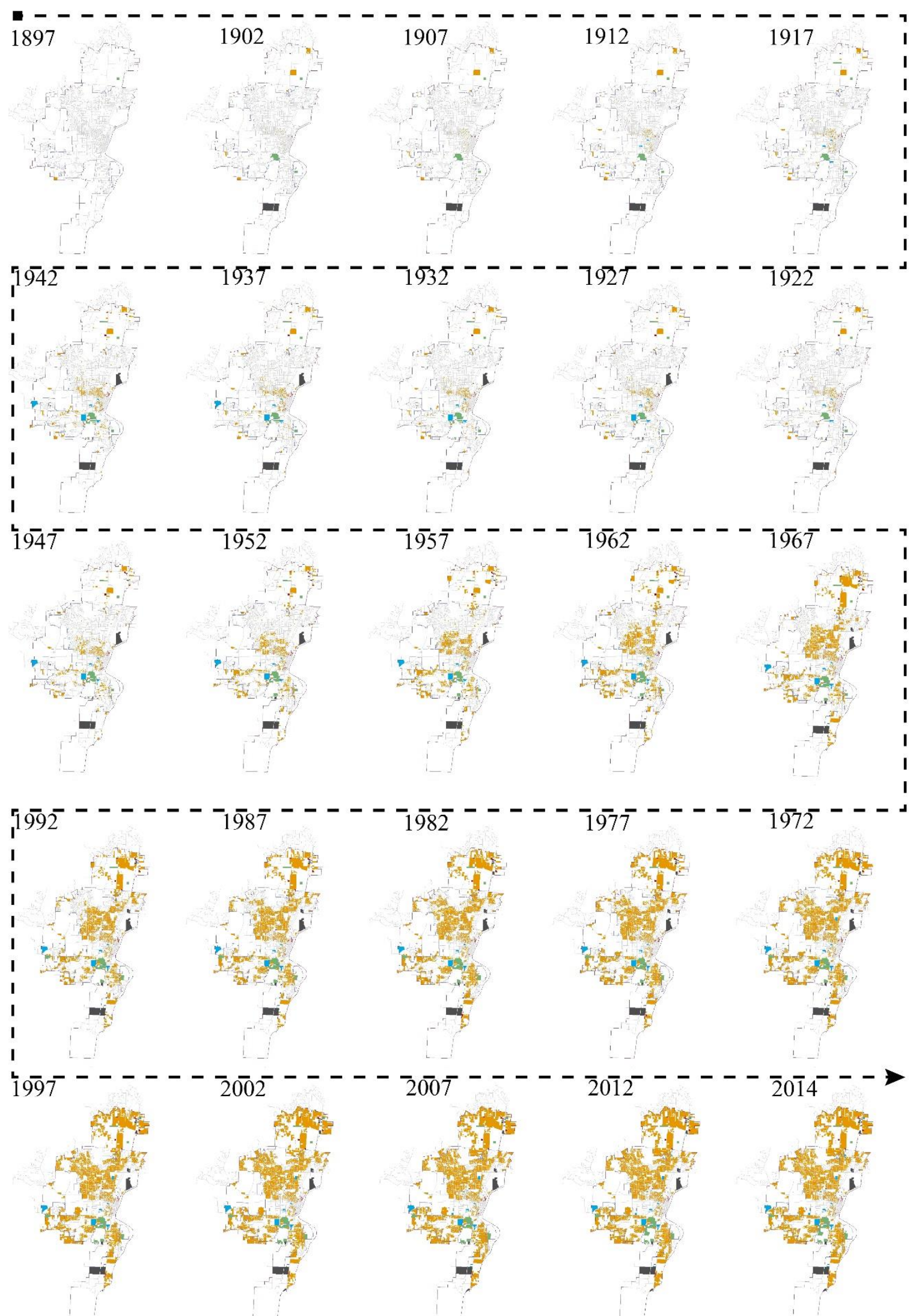

Figure 2. Cumulative Five-Year Urban Growth in Space from 1893 to 2014 Yellow = residence; red $=$ commerce; blue $=$ office; grey $=$ industry, green $=$ open space . 
Table 1 and Figure 3a-h describe the growth of land parcel number, parcel acreage, building square footage, population, and their relative percentages in each historical period. The results show that the growth of land use parcels vary with highs and lows over time. However, several general features can be observed: (1) the growth of the total urbanized parcel, the residential parcel, and the building size have very similar trends, beginning to increase from 1898 to 1902, soaring from 1933 to 1982 and 1988 to 2008, and significantly descending from 1983 to 1987 and 2008 to 2014. This could be attributed to impacts of modern American industrialization and urbanization since the later 19th century [52] and the major economic booms and recessions (i.e., great recession in 1929, early 1980s economy recession [53] and real estate bubble in 2008 [54]). (2) Over these periods, residential land-use consistently accounted for a larger proportion of urbanized lands than any other non-residential land uses. (3) Also, although land parcels vary in sizes, the overall growth patterns in land parcel number and acreage are compatible. (4) Non-residential land uses had some peaks over time, for example, for commercial around 1913-1922 and 2003-2007, industrial in 1898-1902 and 1923-1927, office in 1923-1927, 1933-1937, and 1988-992, and open spaces in 1923-1927.

Table 2 lists 32 variables, which are generally grouped into urban land use and growth $(P, S, R P$, $C P, I P, F P, O P, R S, C S, I S, F S$, and $O S)$, social-economic features ( $L V, I V, A V, M V, B S, P O)$, urban form characteristics $(P D, U D, A S, C M, I M, F M, O M$, and $L M)$, and urban accessibility indicators (CA, IA, $F A, O A$ ). Figure 4 summarizes important cumulative urban growth, land use mix, and value patterns over time periods. These variables, together with their important correlations and regressions, provide good anatomic views of the growth, form, and accessibility of Corvallis.

The average Urban Travel Distance (UTD) from residential land use to office and commercial land uses was growing in a similar pattern from 1918 to 2014 (Figure 4a). This might be attributed to the promotion of retail-office development and construction with concentration in the U.S since the early 20th century [55]. The average UTD from residential land use to industrial land use started to grow from 1938 and was steadily maintained after 1973. The average UTD from residential to open space decreased from 1853 to 1947 and kept increasing from 1948 to 2014, which could be partially explained by the impacts of the City's Beautiful Movement in the early part of the 20th century [56] and the residential suburbanization from the urban core since the middle of 20th century when fast highway expansion and car adoption occurred [57]. The travel distance from residential to industrial and open space were evidently longer than the average accessibility from residential to commercial and office through all time periods. This suggests that the sustainable zoning and development policies in Corvallis have been spatially sound by recognizing the importance of most people living in closer proximity to more frequent destinations, such as workplaces and shops, than to less frequent or specialized areas, such as natural amenities or industrial parks. 
Table 1. Land Use, Size, Urban Form Characteristics by Time Period.

\begin{tabular}{|c|c|c|c|c|c|c|c|c|c|c|c|c|c|c|c|c|c|c|c|c|c|c|c|c|c|c|c|c|}
\hline$t$ & $P$ & $s$ & $R P$ & $C P$ & IP & $F P$ & $O P$ & $R S$ & CS & IS & $F S$ & os & $B S$ & $P O$ & $P D$ & UD & $B S / P$ & $B S / P O$ & $S / P O$ & $P / P O$ & $P / P$ & $S / S$ & $B S / B S$ & $R S / R S$ & $C S / C S$ & IS/IS & $F S / F S$ & OSIOS \\
\hline 53-18 & 2 & 3.27 & 1 & 0 & 1 & 0 & 0 & 49 & 0.00 & 2.78 & 0.00 & 0.00 & 5483 & NA & NA & 1672 & 2728 & NA & NA & NA & $0.01 \%$ & $0.05 \%$ & $0.02 \%$ & $0.01 \%$ & $0.00 \%$ & $0.65 \%$ & $0.00 \%$ & $0.00 \%$ \\
\hline 1858-1862 & 1 & 0.10 & 1 & 0 & 0 & 0 & 0 & 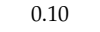 & 00 & 0 & 0.00 & 0.00 & 1602 & NA & NA & 14,564 & 1586 & NA & $\mathrm{N}$ & $\mathrm{N}$ & $0.01 \%$ & $0.00 \%$ & $0.01 \%$ & $0.00 \%$ & $0.00 \%$ & $0.00 \%$ & $0.00 \%$ & $0.00 \%$ \\
\hline 1862-1867 & 0 & 0.00 & 0 & 0 & 0 & 0 & 0 & 0.00 & 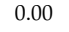 & 0.00 & 0.00 & 0.00 & 0 & NA & NA & 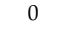 & 0 & NA & NA & NA & $0.00 \%$ & $0.00 \%$ & $0.00 \%$ & $0.00 \%$ & $0.00 \%$ & $0.00 \%$ & $0.00 \%$ & $0.00 \%$ \\
\hline 1868-1872 & 2 & 10.31 & 1 & 0 & 0 & 0 & 1 & 0.31 & 00 & 0.00 & 00 & 10.00 & 5334 & NA & NA & 517 & 2654 & NA & NA & NA & $0.01 \%$ & $0.16 \%$ & $0.02 \%$ & $0.01 \%$ & $0.00 \%$ & $0.00 \%$ & $0.00 \%$ & $1.31 \%$ \\
\hline 1873-1877 & 2 & 0.85 & 2 & 0 & 0 & 0 & 0 & 0.85 & 0.00 & 0.00 & 0.00 & 0.00 & 4341 & $\mathrm{NA}$ & $\mathrm{NA}$ & 5048 & 2160 & NA & $\mathrm{NA}$ & $\mathrm{NA}$ & $0.01 \%$ & $0.01 \%$ & $0.02 \%$ & $0.02 \%$ & $0.00 \%$ & $0.00 \%$ & $0.00 \%$ & $0.00 \%$ \\
\hline 1878-1882 & 4 & 0. & 3 & 0 & 1 & 0 & 0 & & & & & & 621 & & & 10,187 & 1550 & NA & & & $0.03 \%$ & $0.01 \%$ & $0.02 \%$ & $0.01 \%$ & $0.00 \%$ & $0.03 \%$ & $0.00 \%$ & \\
\hline 18 & 1 & 0.0 & 0 & 1 & 0 & 0 & 0 & & & & & & & & 7.50 & 14 & 1. & & & & & 0.0 & $0.01 \%$ & & & & & \\
\hline 18 & 11 & 2.0 & 9 & 2 & 0 & 0 & 0 & 1. & & & 0. & & 18 & & 00 & 94 & 1 & 106 & 0. & & $3 \%$ & $0.03 \%$ & $0.07 \%$ & $0.03 \%$ & $8 \%$ & $0 \%$ & $\%$ & $\%$ \\
\hline 1893-1897 & 18 & 25 & 17 & 0 & 0 & 1 & 0 & 29.09 & & 0.00 & & 0.00 & 31 & 146 & 4.95 & 3 & 17 & 217 & 0 & & $0.13 \%$ & $0.46 \%$ & $0.12 \%$ & $0.58 \%$ & $0.00 \%$ & $0 \% \%$ & $1 \%$ & $\%$ \\
\hline 1898-1902 & 96 & & 83 & 10 & 1 & 0 & 2 & 135.20 & 2.38 & 186.01 & 0. & 78.31 & 169,675 & 635 & & 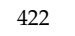 & 1767 & 2 & 0 & & $0.69 \%$ & $6.29 \%$ & $0.67 \%$ & $2.71 \%$ & $6.03 \%$ & $43.54 \%$ & $0.00 \%$ & $10.29 \%$ \\
\hline 1903-1907 & 90 & 88 & 79 & 8 & 3 & 0 & 0 & .02 & 1.15 & 0.71 & 0.00 & 0.00 & 158,264 & 1366 & 57.20 & 6625 & 1758 & 116 & 0.0 & 0. & $0.65 \%$ & $0.37 \%$ & $0.62 \%$ & $0.44 \%$ & $2.91 \%$ & $0.17 \%$ & $0.00 \%$ & $0.00 \%$ \\
\hline $1908-1912$ & 163 & 4 & 150 & 10 & 0 & 2 & 1 & 21 & 1.17 & 0.00 & 9.56 & 5.0 & 13 & 1060 & 15.81 & 4506 & 1853 & 285 & 0.0 & 0. & $1.17 \%$ & $1.05 \%$ & $1.19 \%$ & $1.03 \%$ & $2.96 \%$ & $0.00 \%$ & $5.76 \%$ & $0.66 \%$ \\
\hline & & & & 16 & 1 & 3 & 1 & & & & & & & & & & & & & & & & & & $4.91 \%$ & & & \\
\hline & & & & 12 & 3 & 4 & 1 & & & & & & & & & & & & & & & & & & & & & \\
\hline 19 & 23 & 68 & 21 & 8 & 1 & 3 & 4 & & & 85.43 & 51. & 493.90 & & & & 5 & 1647 & & & & $1.67 \%$ & 1 & $1.50 \%$ & $1.02 \%$ & $7 \%$ & $00 \%$ & $10 \%$ & $64.88 \%$ \\
\hline 1928-1932 & 11 & 55.72 & 104 & 9 & 0 & 2 & 3 & 40 & 1.4 & 0.00 & 0.46 & 13.41 & 210,204 & 71 & 12.76 & 3772 & 1781 & 296 & 0. & & $0.85 \%$ & $0.87 \%$ & $0.82 \%$ & $0.81 \%$ & $3.62 \%$ & $0.00 \%$ & $0.28 \%$ & $1.76 \%$ \\
\hline 1933-1937 & 11 & 67.76 & 111 & 3 & 0 & 1 & 1 & 3 & 0.3 & 0 & 33.12 & 0.5 & 203 & & 5.9 & 300 & 1755 & 5 & & & $0.84 \%$ & $1.06 \%$ & $0.80 \%$ & $0.68 \%$ & $0.81 \%$ & $0.00 \%$ & $19.96 \%$ & $0.08 \%$ \\
\hline & 37 & 126.76 & & 8 & 3 & - & 5 & & & & & 77 & & & 14 & 48 & 16 & & & & & & $2.42 \%$ & & $8.74 \%$ & & $1.06 \%$ & \\
\hline & & & & 6 & 3 & 6 & 7 & & & & & & & & & & & & & & & & & & $87 \%$ & & & \\
\hline & & & & 3 & 13 & & & & & & & & & & & & & & & & & & & & & & & \\
\hline $1953-19=$ & 75 & & 7 & 3 & 2 & 0 & 1 & & & & & 2 & & & & & & & & & & & & & & $\%$ & & $0.27 \%$ \\
\hline $1958-19$ & 11 & & 11 & 2 & 8 & 3 & 4 & & 1. & 4. & & 10 & & & & & 16 & & & & & 5. & 7.5 & $6.68 \%$ & $\%$ & $\%$ & $\%$ & $1.36 \%$ \\
\hline & & & & 3 & 16 & 1 & 2 & & 1. & & & 0. & & & & 30 & & & & & & & & & $4.15 \%$ & & & $0.11 \%$ \\
\hline 19 & & & & 3 & 8 & 0 & 3 & & & & & & & & & & & & & & & & & $6.14 \%$ & & $0.91 \%$ & & \\
\hline 19 & 13 & & & 0 & & 4 & 1 & & & 13. & & & & & & & & & & & & 7. & $9.03 \%$ & $\%$ & $\%$ & $\%$ & $\%$ & $0.32 \%$ \\
\hline & 12 & & 12 & 1 & 1 & 1 & 0 & & & & & & & & & & & & & & & & & & & & & \\
\hline & 24 & & & - & & & & & & & & & & & & & & & & & & & & & & & & $\%$ \\
\hline & 60 & & 0 & 0 & 0 & 2 & 2 & & & & & 1. & & & & & & & & & & & & & & & & \\
\hline & 10 & & & 0 & 1 & 1 & 1 & & & & & & & & & & & & & & & & & & & & & \\
\hline & 88 & & & 1 & 1 & 0 & 0 & & & 10 & & & & & & & & & & & & & & $4.94 \%$ & & $2.41 \%$ & & $0.00 \%$ \\
\hline 2003-2007 & 1189 & 301.54 & 1155 & 30 & 0 & 2 & 2 & 285.36 & 1.1 & 0.00 & 6.37 & 8.62 & $2,594,679$ & 2159 & 7.1 & 8604 & 2182 & 1202 & 0. & 0. & $8.57 \%$ & $4.72 \%$ & $10.18 \%$ & $5.71 \%$ & $3.01 \%$ & $0.00 \%$ & $3.84 \%$ & $1.13 \%$ \\
\hline & 207 & 135.2 & 193 & 1 & 1 & 11 & 1 & & 0. & & & 41 & & 2861 & 21.15 & 27 & 24 & 179 & 0. & & & $2.12 \%$ & $2.00 \%$ & $1.80 \%$ & $1.16 \%$ & $0.23 \%$ & $1.39 \%$ & $5.48 \%$ \\
\hline & 02 & & 7. & & & 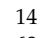 & 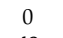 & & & & & & & & & & & & & & & & & & & & & \\
\hline & & 8.90 &, 553 & 140 & 70 & 69 & 48 & 994.92 & $39.4 \mathrm{c}$ & 7.24 & & & 9,07 & 3,74 & & 990 & & 474 & 0.12 & 0.26 & & & & $0 \%$ & $0 \%$ & $0 \%$ & $10 \%$ & \\
\hline
\end{tabular}


Table 2. Cumulative Urban Growth, Form, Socio-Economic, and Accessibility Values.

\begin{tabular}{|c|c|c|c|c|c|c|c|c|c|c|c|c|c|c|c|c|c|c|c|c|c|c|c|c|c|c|c|c|c|c|c|}
\hline \multirow{3}{*}{$\begin{array}{c}\text { Period } \\
t\end{array}$} & \multicolumn{12}{|c|}{ Urban Land Uses and Growth } & \multicolumn{7}{|c|}{ Social Economics Features } & \multicolumn{7}{|c|}{ Urban Form Characteristics } & \multirow{2}{*}{\multicolumn{5}{|c|}{$\begin{array}{l}\text { Urban Accessibility Measure } \\
\text { Urban Travel Distance (UTD) }\end{array}$}} \\
\hline & Parcel & Size & & d Use & Parc & el No & & & nd Us & se Parc & cel Size & & & Valu & & & Bldg & Populat & ion & Densi & & & & Land Use Mi & & & & & & & \\
\hline & $P$ & $s$ & $R P$ & $C P$ & $I P$ & $F P$ & $O P$ & $R S$ & CS & IS & $F S$ & $O S$ & $L V$ & $I V$ & $A V$ & $M V 1$ & $B S$ & $P O$ & $P D$ & UD A & AS & $C M$ & $I M$ & $F M$ & $O M$ & $L M$ & $C A$ & $I A$ & $F A$ & $O A$ & $T A$ \\
\hline 853-1897 & 41 & 47 & 34 & 3 & 2 & 1 & 1 & 33 & 0.4 & 2.9 & 0.4 & 10.0 & 0 & 5 & T & 10 & 7 & 1,731 & 37.07 & 70.161 & 1.14 & $\times 10$ & $\times 10$ & $\times 10$ & $\times 10$ & $8 \times 10>$ & 6.2 & 16.2 & 5.1 & 37.4 & 65.0 \\
\hline 100 & 137 & 449 & 117 & 13 & 3 & 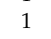 & 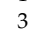 & 168 & 2.7 & 188.9 & 0.4 & 88.3 & 22 & 15 & . & 37 & 24 & & 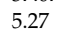 & 0.053 & 3.27 & - & & & (1) & & 13.0 & .0 & 5.5 & 3.6 & 1.0 \\
\hline 19 & 227 & 472 & 196 & 21 & 6 & 1 & 3 & 190 & 3.9 & 189.6 & 0.4 & 88.3 & 34 & 24 & 39 & 58 & 40 & & 7.90 & 0.092 & 2.08 & $2.3 \times 10^{-4}$ & $7 \times 10^{-4}$ & -5 & $7 \times 10^{-5}$ & $\times 10^{-4}$ & 11.4 & 16.6 & 1 & 5 & 56.5 \\
\hline-1912 & 390 & 540 & 346 & 31 & 6 & 3 & 4 & 242 & 5.1 & 189.6 & 10.0 & 93.3 & 61 & 51 & 80 & $112 ?$ & 70 & 4792 & 8.88 & 0.131 & 1.38 & $1.7 \times 10^{-4}$ & $3 \times 10^{-4}$ & $\times 10^{-5}$ & $3.0 \times 10^{-5}$ & $5 \times 10^{-4}$ & 9.9 & 16.4 & 5.6 & .1 & 53.0 \\
\hline & 563 & 600 & 498 & 47 & 7 & 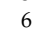 & - & 276 & 7.0 & 189.7 & 17.4 & 109.8 & 86 & 75 & 111 & 161 & 101 & & 8.98 & 0.171 & 1.07 & $1.6 \times 10^{-4}$ & $6 \times 10^{-4}$ & & $1.8 \times 10^{-5}$ & $3.6 \times 10^{-4}$ & 8.3 & 14.6 & 7.8 & 1.2 & 51.8 \\
\hline & 7 & 67 & 68 & 59 & 10 & 10 & 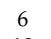 & se & 99 & 194.0 & 19 & 113.1 & 116 & 97 & 144 & 214 & 13 & & 6 & 0.200 & 0.88 & & & & & & & & & & 2 \\
\hline $3-1927$ & 1000 & 1359 & 89 & 67 & 11 & 13 & 10 & 386 & 15.3 & 279.4 & 70.6 & 607.0 & 238 & 124 & 249 & 361 & 174 & & 5.18 & 0.131 & 1.36 & & & & & & 9.1 & 7 & & & \\
\hline 1853-1932 & 1118 & 1414 & 1003 & 76 & 11 & 15 & 13 & 427 & 16.8 & 279.4 & 71.1 & 620.4 & 254 & 137 & 269 & 391 & 195 & 7746 & 5.48 & 0.141 & 1.27 & 5. & -5 & -5 & $1.2 \times 10^{-5}$ & $\times 10^{-4}$ & 9.6 & 14.9 & 9.3 & 5.3 & 49.1 \\
\hline 1853-1937 & 12 & 14 & 1114 & 79 & 11 & 16 & 14 & 461 & 17.1 & 279.4 & 104.2 & 621.0 & 270 & 154 & 292 & 4242 & 215 & 81 & 5.50 & 0.151 & 1.20 & -5 & & -5 & $1.0 \times 10^{-5}$ & $10^{-4}$ & 9.6 & 15.0 & 10.3 & 15.3 & 50.2 \\
\hline & 16 & & 1466 & 87 & 14 & 21 & Ne $>$ & 573 & 20.5 & 280.7 & 106. & 628.7 & 322 & 19 & 355 & 5142 & 277 & 9 & 6.19 & 0.171 & 1.003 & & & & & & 7 & 4 & & & 2.6 \\
\hline & & & & 93 & 17 & 27 & s & & & & & & & & 0 & & 338 & & & & & & & & & & & & & & \\
\hline 1853-1952 & 2916 & 2243 & 2729 & 96 & 30 & 30 & 31 & 1117 & 27.9 & 333.6 & 108.8 & 656.4 & 499 & 319 & 582 & 818 & 484 & 17,099 & 7.62 & 0.220 & 0.77 & $1.6 \times 10^{-5}$ & $0^{-5}$ & $10^{-6}$ & $3.9 \times 10^{-6}$ & $\times 10^{-5}$ & 2.7 & 20.0 & 11.6 & 4.9 & 59.2 \\
\hline 1957 & 3673 & & 3480 & 99 & 32 & 30 & 32 & 1 & 29. & 375.0 & 108. & 658.5 & 606 & 407 & 737 & $1012 \epsilon$ & 617 & 19,330 & 7.39 & 0.240 & 0.71 & $1.1 \times 10^{-5}$ & & & $2.5 \times 10^{-6}$ & $10^{-5}$ & 2.7 & 20.6 & 1 & 5.0 & 60.4 \\
\hline & & & & & 40 & & & & & & & & & & & & & & & & & & & & & & & & & & \\
\hline & & & 56 & 10 & 56 & 34 & 38 & & & & & & & 617 & 1180 & & & & 4 & & & & & & & & & & & & \\
\hline & 69 & & 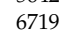 & 10 & 64 & 34 & 41 & & 8 & 387.8 & 112.7 & 683.4 & 1040 & 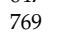 & 1395 & & 1178 & & & 0.300 & 0.56 & & & & & & & 3 & & & \\
\hline & 82 & & 80 & 10 & 66 & 38 & $\begin{array}{l}11 \\
42\end{array}$ & & 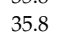 & 401.4 & 12 & 68 & 12 & 92 & 16 & 21 & & & & 0.320 & 0.52 & & & & & & 5.5 & .7 & & & 16 \\
\hline & & & 9330 & 10 & 67 & 39 & 2 & & 37.7 & 406.4 & 128 & 685.9 & 1359 & & 19 & 24461 & & & & 0.340 & 0.49 & & & & & & 6.1 & 21.1 & .7 & 6 & .6 \\
\hline & & 48 & 年 & 10 & 67 & 39 & 42 & & $37:$ & 406.4 & & & 1396 & & & 2521 & & & & 0.340 & & & & & & & 6.2 & 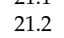 & & & \\
\hline & 10 & & 10 & 10 & 67 & 41 & - 18 & & 37 & 40 & & & & & & & & & & 0 & 0.50 & & & & & & & & & & \\
\hline & & & & 10 & 68 & 42 & 45 & & 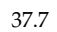 & & & & & & & & & & & 0 & 0.49 & & & & & & & .6 & & & \\
\hline & & 5913 & & 109 & 69 & 42 & 45 & 458 & 37.8 & 426.2 & 15 & 710.9 & 17 & & & 33 & & & 0 & 0.370 & 0.48 & & & & 3.0 & $2.8 \times 10^{-6}$ & 17.1 & 22.1 & 17.1 & 9.2 & 5.4 \\
\hline & & & & 139 & 69 & 44 & 47 & 196 & re $>$ & & & & 1000 & & & & & & & & 0.46 & & & & & & - & .4 & 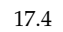 & 1 & 76.4 \\
\hline & & & & 14 & 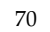 & 55 & 48 & & 39 & & & & & & & & & & & & & & & & & & & & & & \\
\hline 014 & & 6389 & 13,553 & 140 & 70 & 69 & 48 & 4995 & 39.5 & & & & & & & & & & 8.60 & & 0.46 & $\times 10$ & $\times 1$ & $7 \times 16$ & $.6 \times 10$ & $\times 10^{-6}$ & 17.8 & 22.6 & 17.8 & 19.1 & 7.4 \\
\hline
\end{tabular}




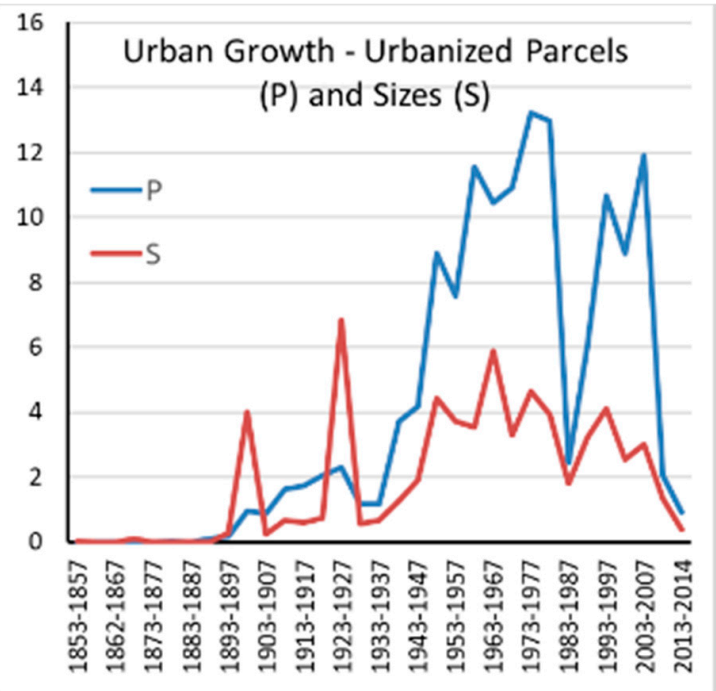

(a)

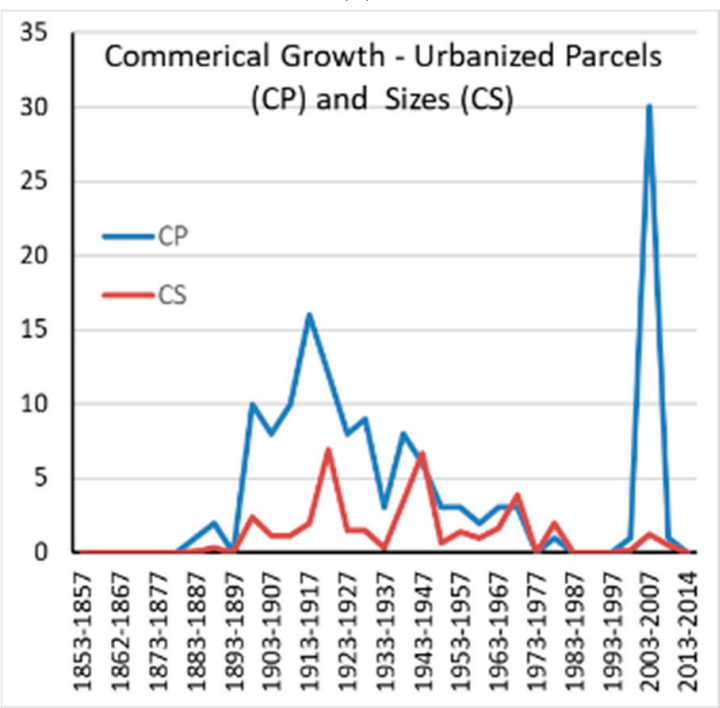

(c)

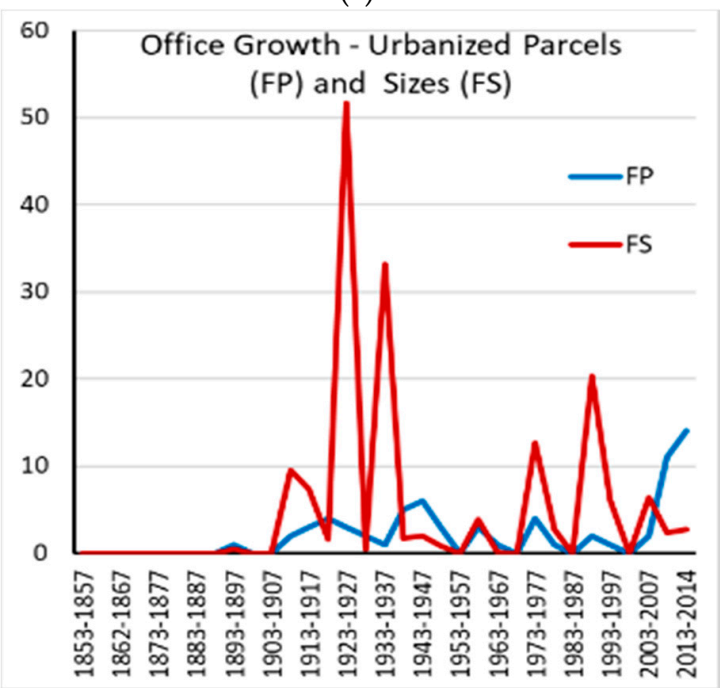

(e)
Residential Growth - Urbanized Parcels (RP) and Sizes (RS)

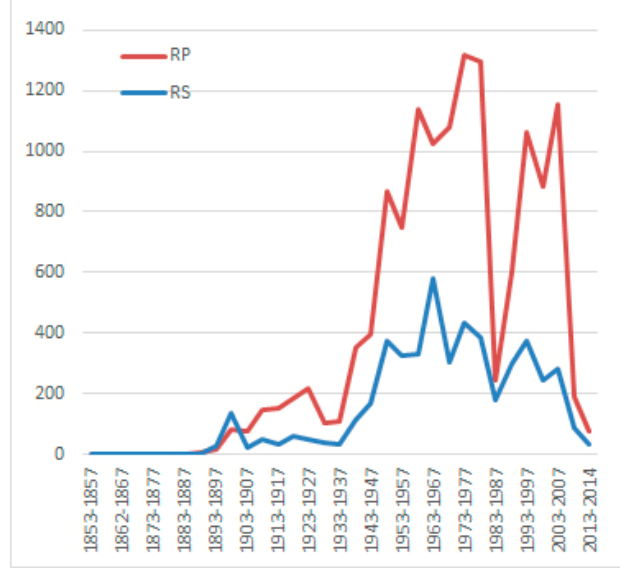

(b)

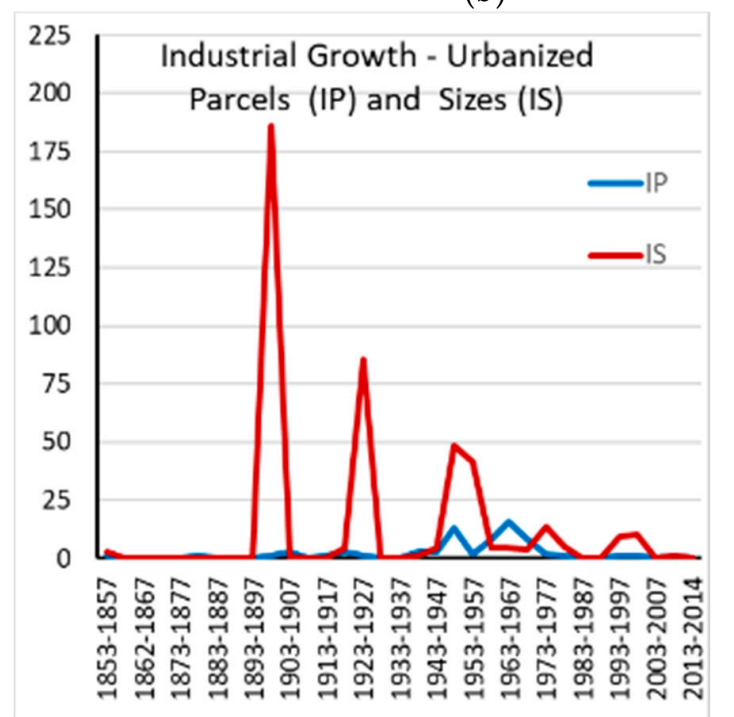

(d)

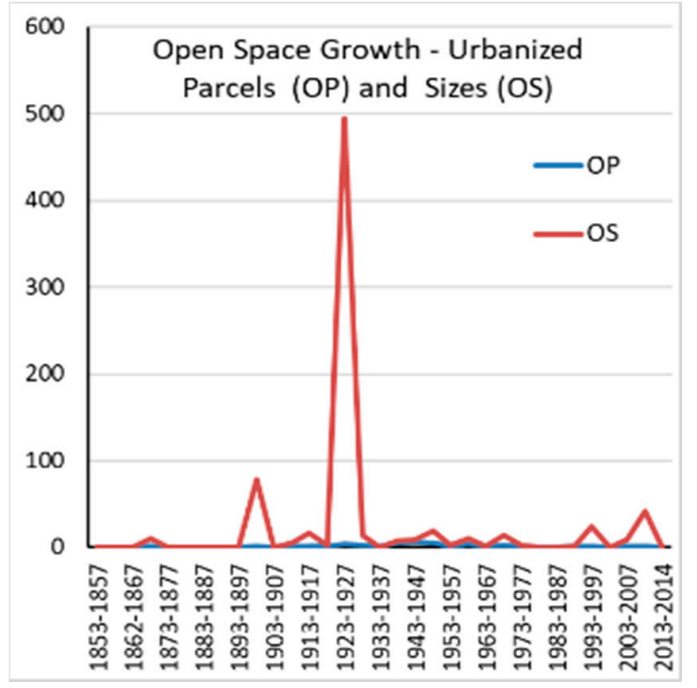

(f)

Figure 3. Cont. 


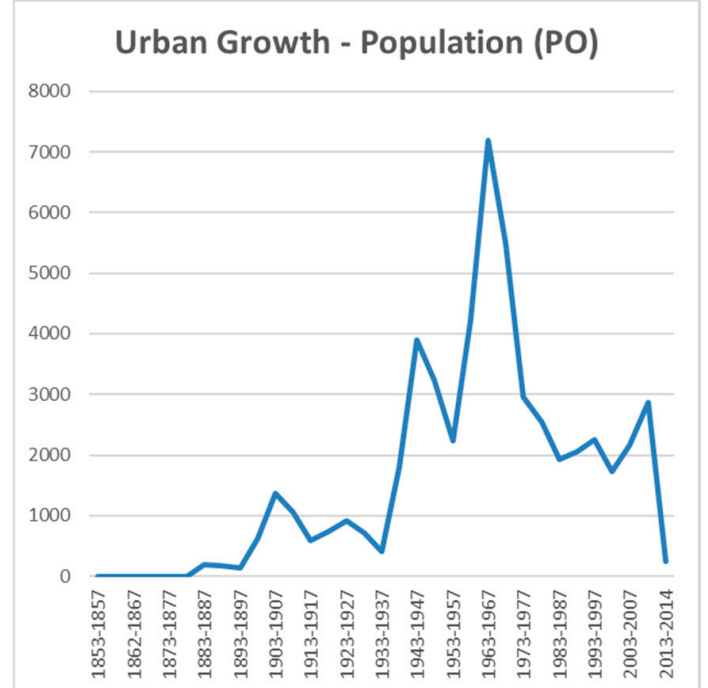

(g)

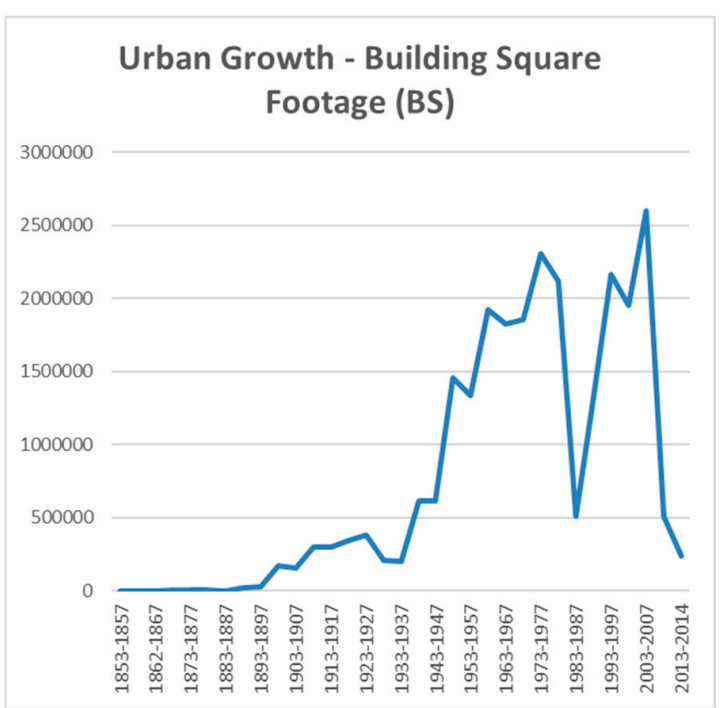

(h)

Figure 3. Urban Growth by Time Period. (a) Urban Growth; (b) Residential Land Use Growth; (c) Commercial Land Use Growth; (d) Industrial Land Use Growth; (e) Office Land Use Growth; (f) Open Space Land Use Growth; (g) Population Growth; (h) Building Square Footage Growth.

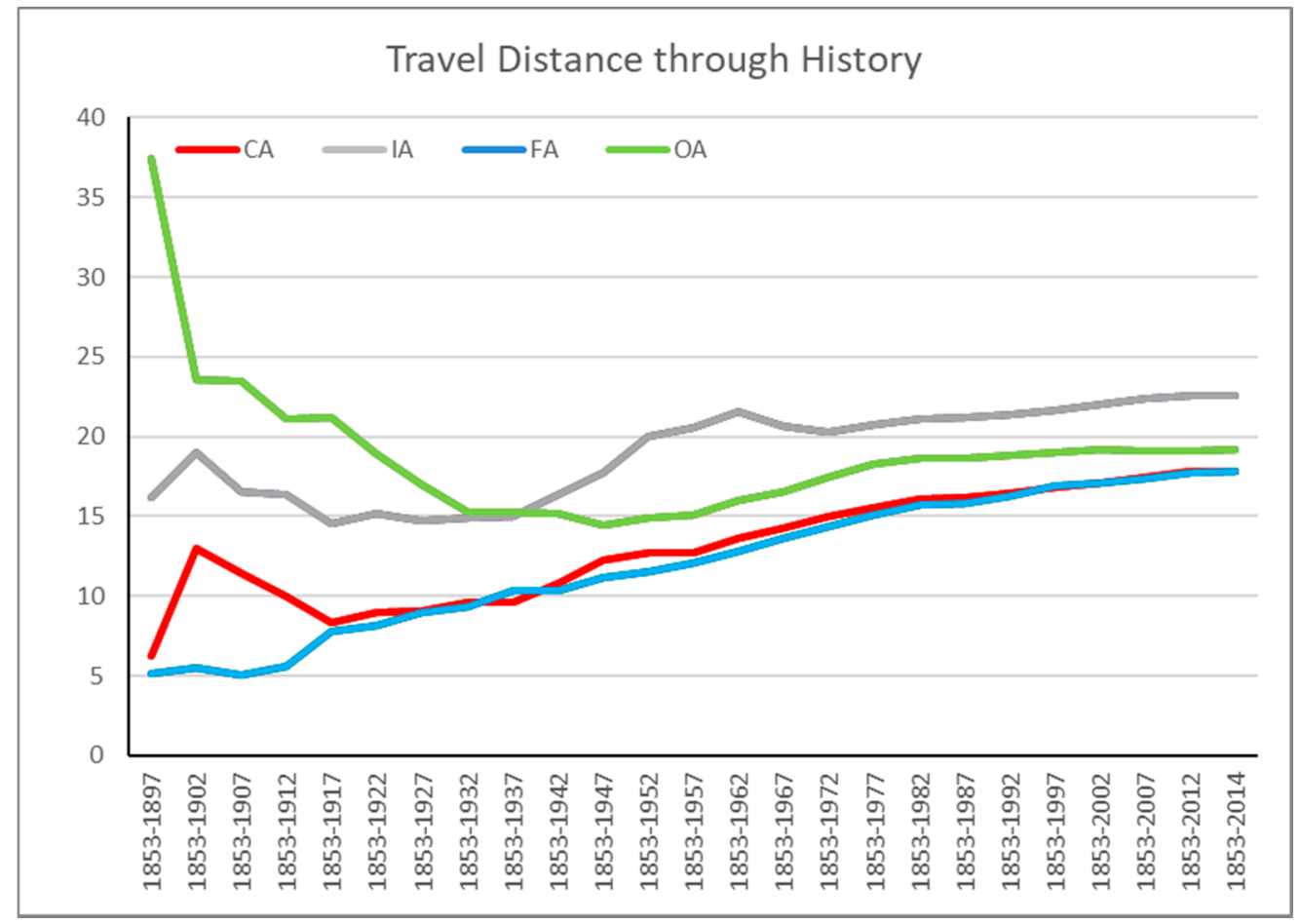

(a)

Figure 4. Cont. 


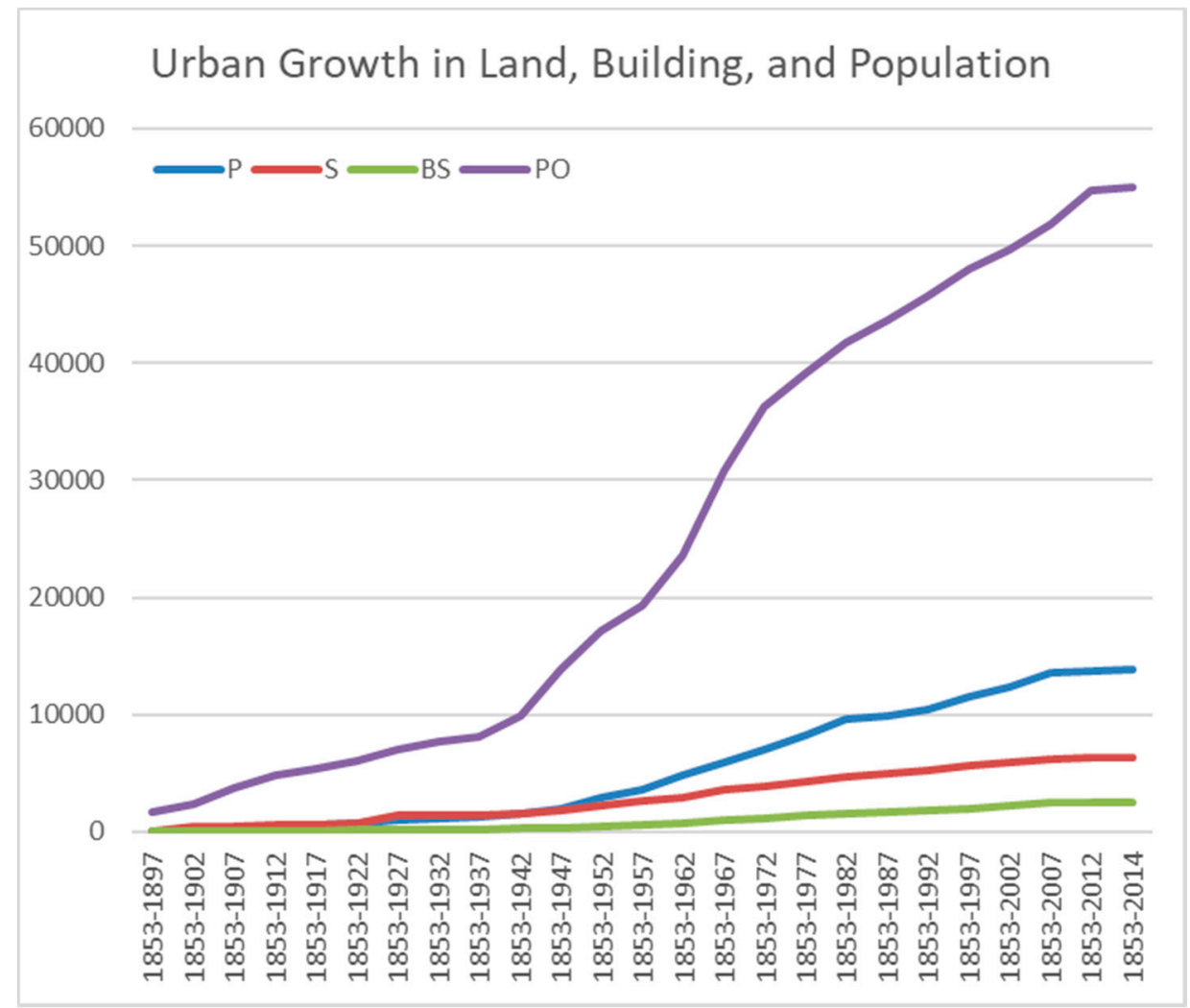

(b)

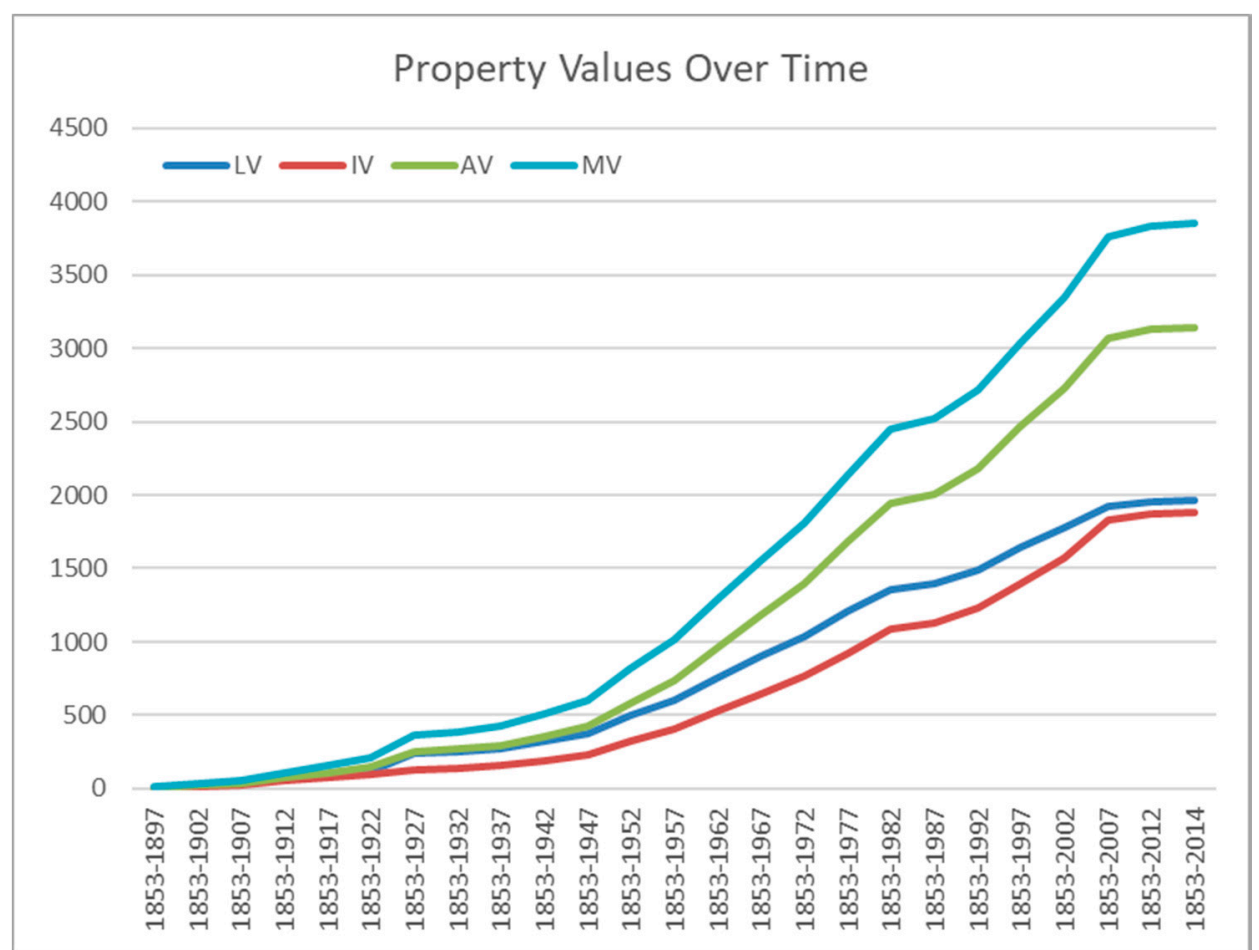

(c)

Figure 4. Cumulative Urban Growth Patterns and Accessibility Changes. (a) UTD through History; (b) Urban Growth in Land, Building and Population; (c) Property Values over Time. 
Figure 4a also shows that accessibilities indicated by urban travel distances to commercial, industrial, office, and open space land (CA,IA, FA, OA) experienced both ups and downs until 1967, indicating a few new parcels would change the accessibility significantly early on. However, the urban travel distances stabilized and steadily increased (accessibility decreased) over time, especially after 1932. These could be explained by the facts that people resided in isolation in the 19th century and since the beginning of the 20th century industrialization and urbanization, especially the auto use and highway network, had expedited the shift from agricultural activities to urban lifestyles and formed the infrastructure of modern American cities [52,58,59]. Figure $4 \mathrm{~b}$ indicates the population as a slight S-curve, 1853-1937 and 1972-2012 are two large periods with steady increase, but at a rate lower than the period of 1937-1972, in which the United States entered two world wars and enjoyed subsequent post-war economic prosperity. These slow, moderate, and fast growth periods in population were reflected in land parcel, land size, and building space growth in the same periods. This might be due to the fact that population increases require more residential lands and residential land-use accounts for a larger proportion of urban land than any non-residential land-uses throughout history [60]. The average land size is generally decreasing with sharp increases during the 1897-1902 and 1922-1927 periods. Figure $4 \mathrm{c}$ shows an ever-increasing property value trend, regardless land value, improved value (building value), assessed value, and market value, indicating Corvallis is attractive for real estate investment. Also, although an assessed value (by government) and its corresponding market value (by the market) both include a building and land value for a property, the assessed value is always lower than the market value and the difference has been increasing in Corvallis. Since the property tax and homestead reduction are all based upon assessed value in Oregon, residents in Corvallis are better off if the market value is higher than the assessed value. However, the land value $(L V)$ being consistently higher than the improved value $(I V)$, even if they have been converging since 2007, indicates that Corvallis's land market is more active and expensive than its housing market.

\subsection{Relationships between Urban Growth, Accessibility and Form}

Figure 5 is a14 $\times 14$ scatterplot matrix showing the correlations among variables of urban form and growth by land use (i.e., parcel, size, and population) over the historical time periods. Scatterplot matrix is a useful visualization tool for quick exploratory analysis of common statistical relationships. Most variables are not correlated with points out-spreading (i.e., $O P$ vs. all others) or seemingly linear yet non-corresponding (i.e., $O S$ vs. all others) or concentrated with outliers (i.e., $I P$ vs. all others). Only a few strong correlations and linear relationships exist between $P, S, R P, R S, B S, P O$ (i.e., ranging from $R^{2}=0.64$ for $S$ vs. $R S$ to $R^{2}=0.995$ for $P$ vs. $R P$ ). There are positive correlations between the population growth and the total urbanized parcel number $\left(R^{2}=0.58\right)$, the total residential land acreage $\left(R^{2}=0.71\right)$, and the total building square footage $\left(R^{2}=0.52\right)$. These indicate and confirm that population growth and the residential housing needs are the primary reasons for the overall urbanization in this city.

The number of the residential parcels developed in each period is highly and positively linear-correlated with the total residential parcel size $\left(R^{2}=0.86\right)$, but this kind of parcel number vs. parcel size correlation is not clear for commercial, industrial, office, and open space parcels. This suggests that in Corvallis, the demand for residential land use is relatively stable and responsive to the population growth without many highs and lows while the land demand for other social, economic, or technological needs is less responsive to population growth. In other words, commercial, office, or industrial land uses such as shopping malls, office complex, and the industrial parks are often for a longer-term and more cyclical and variant in development [61,62].

Does this mean that Corvallis's urban growth in non-residential land uses was random or not coordinated in public urban planning and private land development? The sizes and parcels for non-residential land uses themselves seems to suggest so. Yet, a closer look at all of them together over the time periods yields a quite different view. Figure 6 shows the percentages of different land use development with respect to their cumulative totals by 2014 at different time periods. Apparently, 
different land uses were developed during similar time periods. For example, 1898-1902 and 1923-1927 periods saw all five land uses. Other periods had at least some different land uses, with the rest either ahead of or falling behind a couple of time periods.

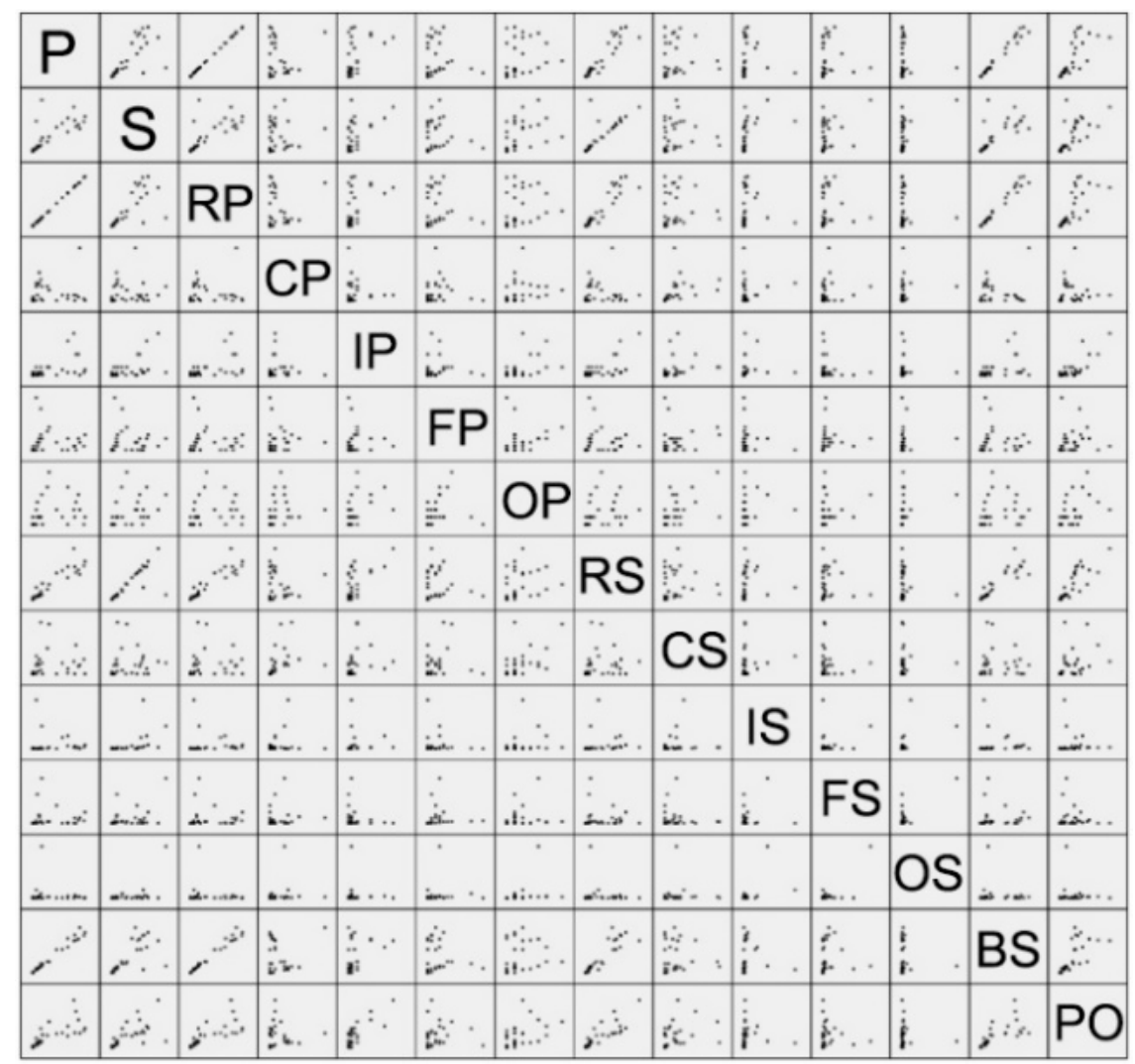

Figure 5. Correlation Matrix of Land Use Parcel, Size, and Population by Time Period.

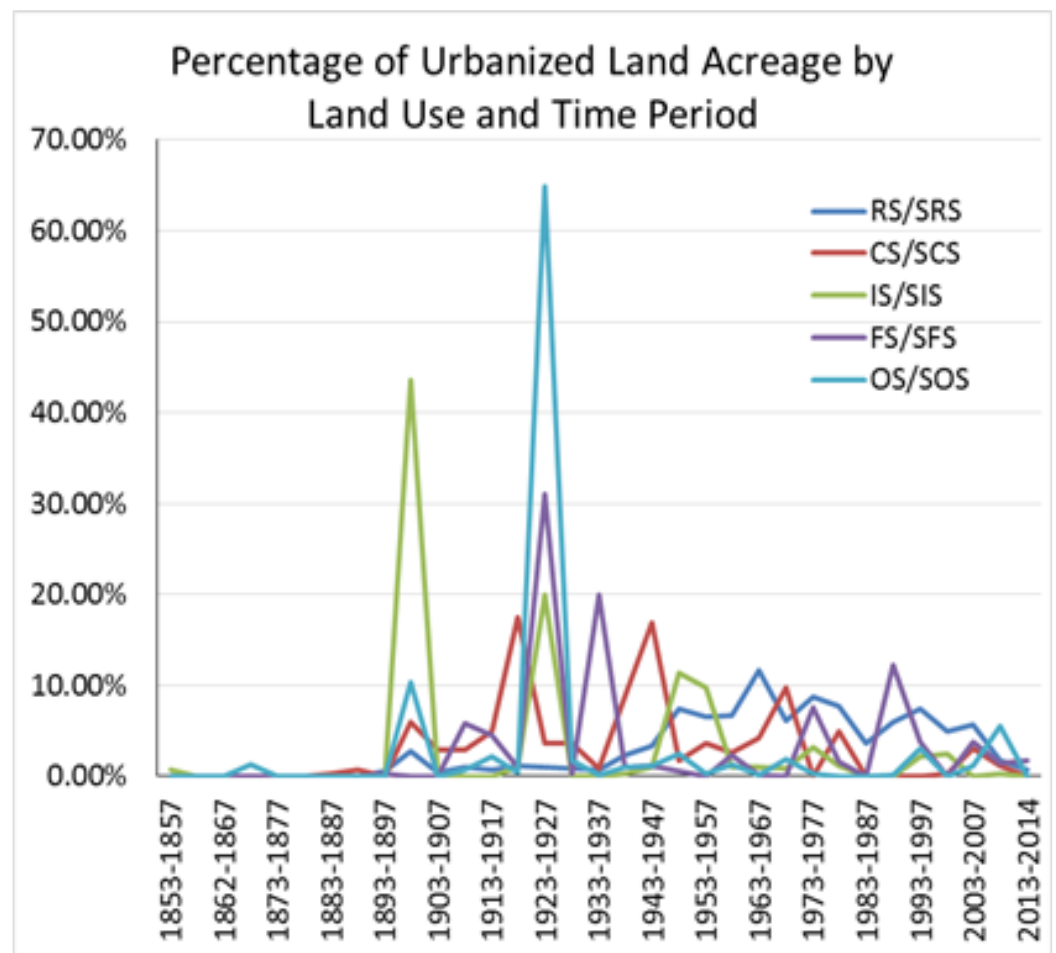

Figure 6. Development Synchronicity of Different Land Uses. 
Figure $7 \mathrm{a}, \mathrm{b}$ are two scatterplot matrixes showing the correlations between the 32 variables. Visually, it is clear that most correlations are strong and apparent with only a few weak or no correlations. Specifically, five strong correlations exist:

(1) linear or close to linear relationship (i.e., $B S$ or $P O$ vs. $P, S$, and $R P ; B S$ vs. $L V, I V$, and $A V$ in Figure $7 \mathrm{a} ; P, S$, and $R P$ vs. $L V, I V, A V$, and $M V$, and between $L V, I V, A V$, and $M V$ in Figure $7 \mathrm{~b}$ ).

(2) exponential or power relationship (i.e., $C A, L V, I V, A V, M V$ vs. $U D, I M, F M$, and $S M$ or vs. $P, S$, $R P$ in Figure $7 \mathrm{a}$ and $P, S$ vs. $A S, C M, I M, F M$ in Figure $7 \mathrm{~b})$.

(3) logarithmic relationship (i.e., $F A$ or $U D$ vs. $P, S$, and $R P$, or vs. $L V, I V, A V$, and $M V$ in Figure 7 a and $C P, I P, F P$, and $O P$ vs. $L V, I V, A V$, and $M V$ and $C P$ vs. $I P, F P, O P$, and $R S$ in Figure $7 \mathrm{~b}$ ).

(4) moving average relationship (i.e., $C A, I A, F A$, and $O A$ vs. all other variables except $O S$ in Figure $7 \mathrm{a}$ and $O A, T A$ vs. $P, S, C M, F M, O M, L M$ in Figure $7 b)$.

(5) no relationship (i.e., $O S$ or $P D$ vs. all other variables in Figure 7 a and $P D$ vs. $U D, A S, C M$, IM, $F M, O M, L M, I A, F A, O A$, and $T A$ in Figure $7 \mathrm{~b}$ ). It is interesting to see that some correlations seem to be bi-modal (i.e., $O S$ vs. all accessibility or IS or $F S$ vs. some accessibility in Figure 7a and $O S$ vs. parcels and $O S$ vs. all values in Figure $7 \mathrm{~b}$ ). Some representative strong correlation patterns are illustrated as enlarged charts in Figure 8, labeled with linear or non-linear $R$-square values.

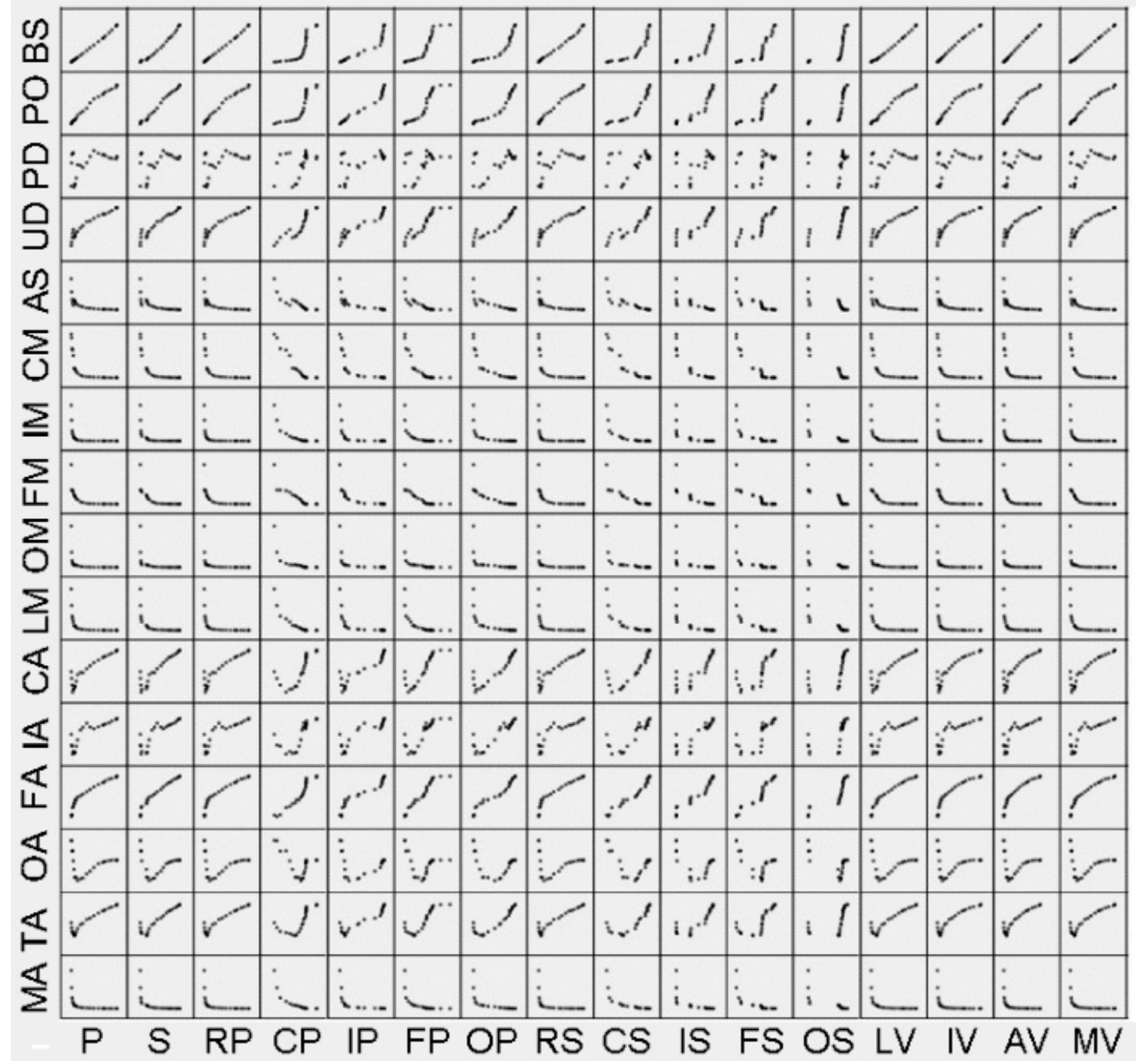

(a)

Figure 7. Cont. 


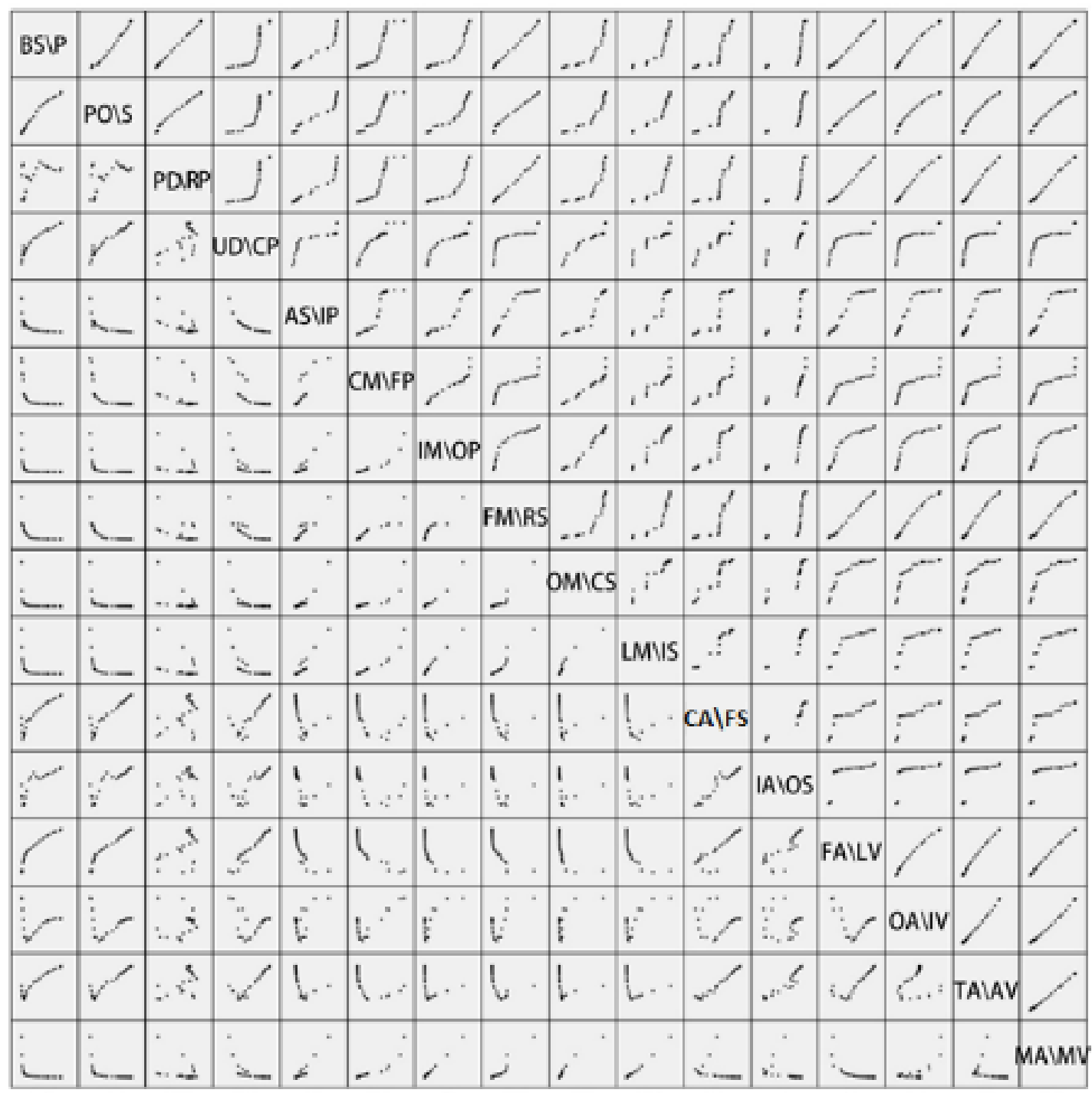

(b)

Figure 7. Correlations for 32 Urban Growth, Form, Accessibility, and Socio-Economic Variables. (a) scatterplot matrix of ( $B S, P O, P D, U D, A S, C M, I M, F M, O M, L M, C A, I A, F A, O A, T A, M A)$ vs. ( $P, S$, $R P, C P, I P, F P, O P, R S, C S, I S, F S, O S, L V, I V, A V, M V)$; (b) scatterplot matrix between ( $B S, P O, P D, U D$, AS, CM, IM, FM, OM, LM, CA, IA, FA, OA, TA, MA) and (P, S, RP, CP, IP, FP, OP, RS, CS, IS, FS, OS, LV, $I V, A V, M V)$.

In Figure 8a, the urbanized parcel number is strictly linearly correlated with the population $\left(R^{2}=0.99\right)$, which confirms that the urban development in Corvallis corresponds sensitively with the population growth through the City's history. As the City grows with more urbanized parcels, the building square footage density increases correspondingly $\left(R^{2}=0.68, R^{2}=0.91\right.$ respectively) in Figure $8 \mathrm{~b}$. The commercial parcel numbers increase with the growing of the total residential parcel size $\left(R^{2}=0.85\right)$ in Figure $8 \mathrm{c}$. The UTD to commercial linearly increases along with the building space density in Figure 8d. However, the average UTD from residential parcels to commerce office, and industrial parcels show strong non-linear relationships with the land-use mixes, decreasing quickly then slowing down through most of the City's history, in Figure 8e-h. This indicates that increasing the land uses mix degree is more effective for reducing urban travel distance and thus improving accessibility than increasing urban density. 


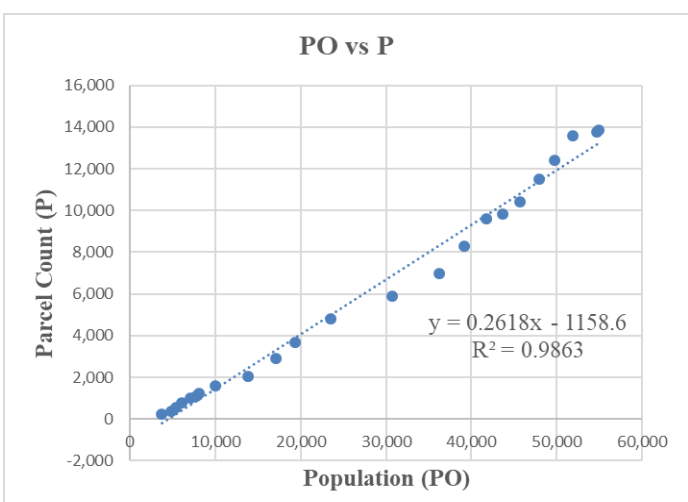

(a)

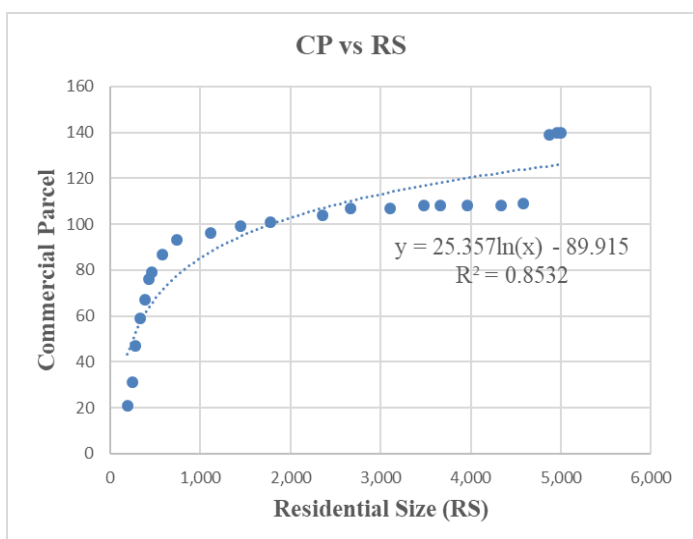

(c)

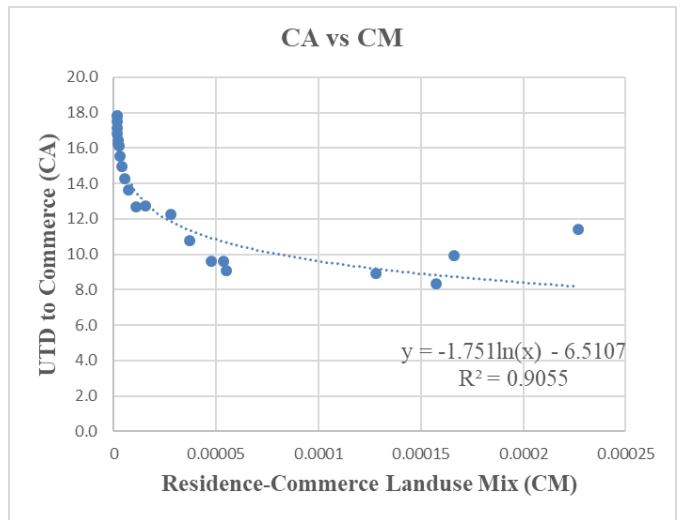

(e)

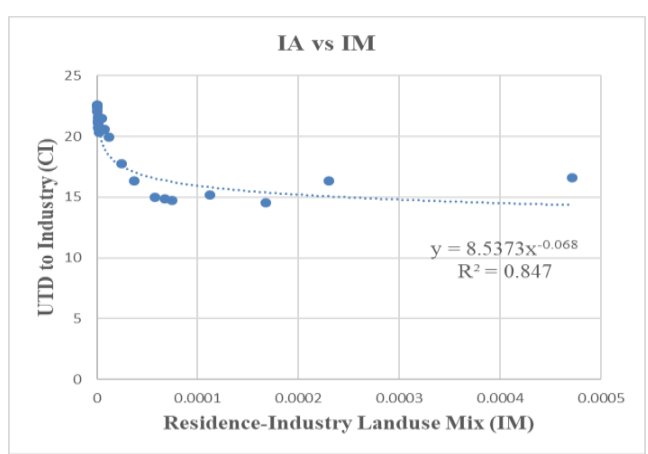

(g)

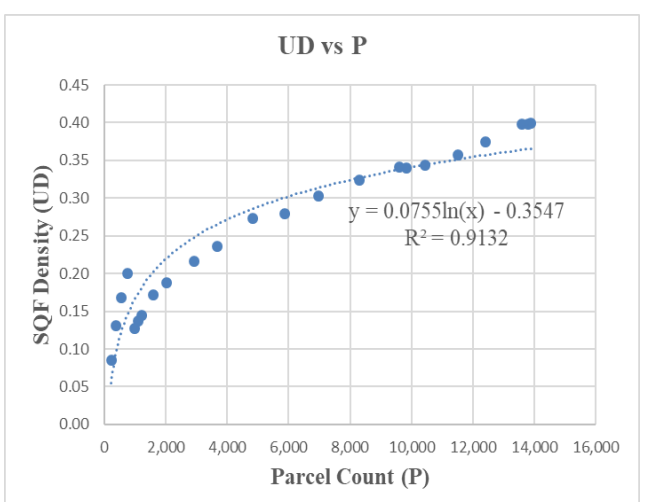

(b)

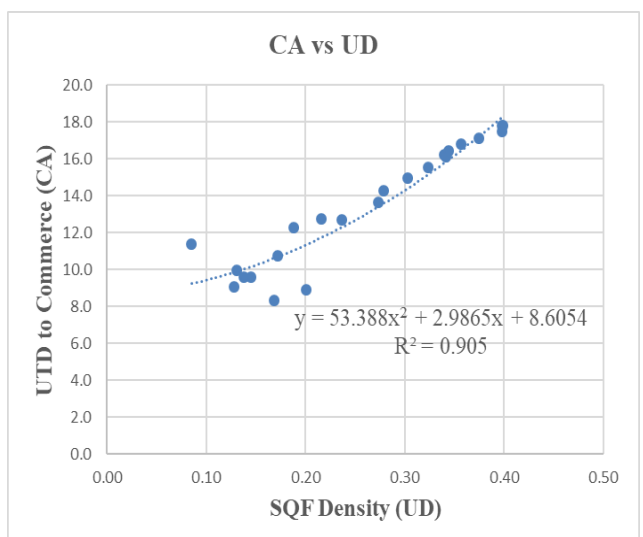

(d)

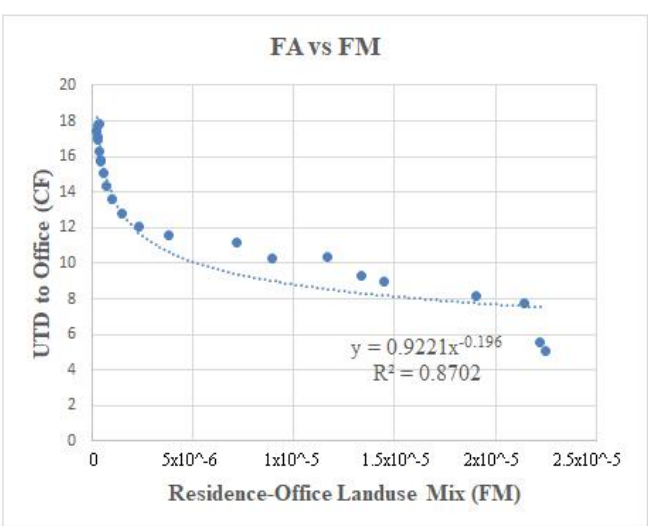

(f)

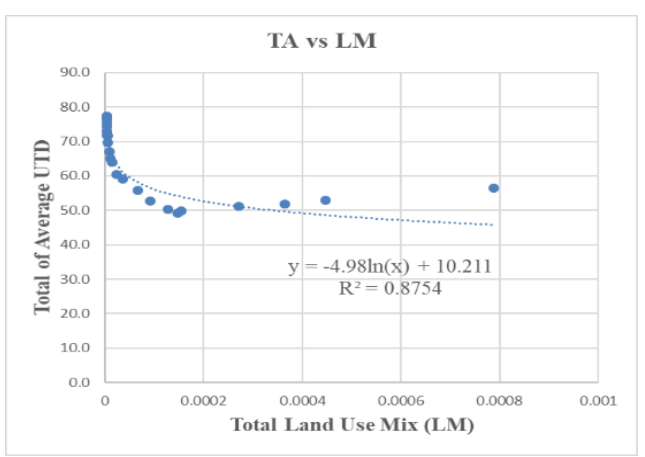

(h)

Figure 8. Cont. 


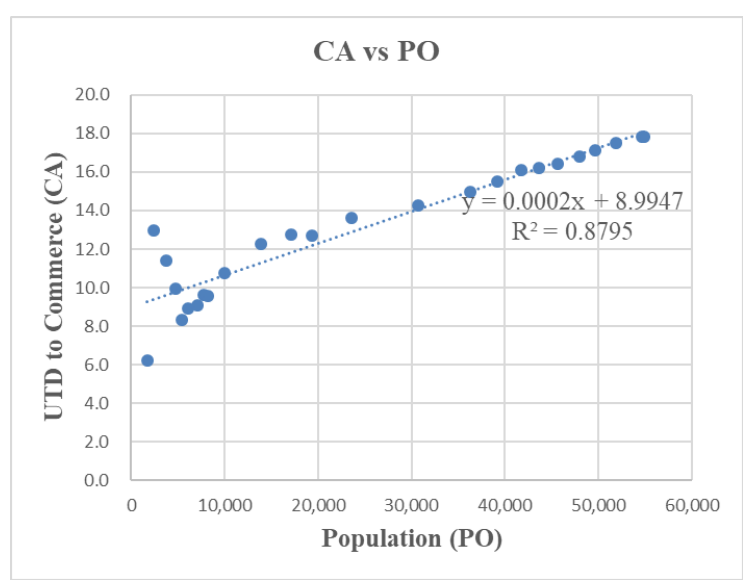

(i)

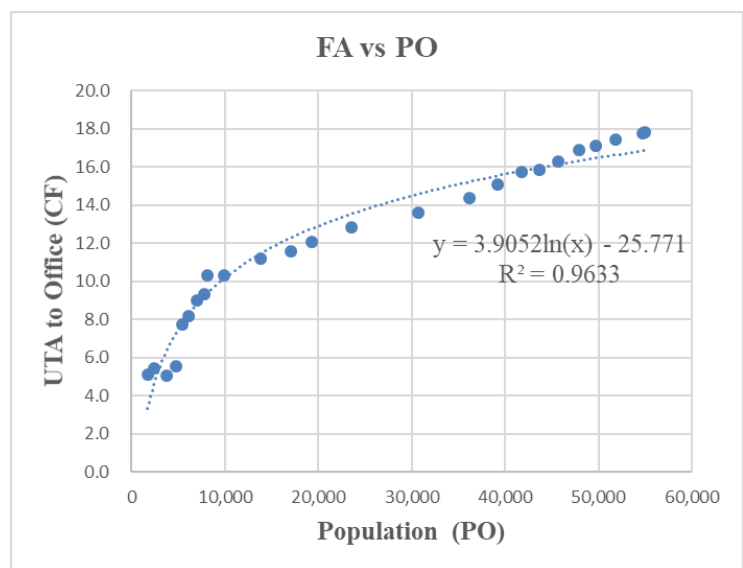

(k)

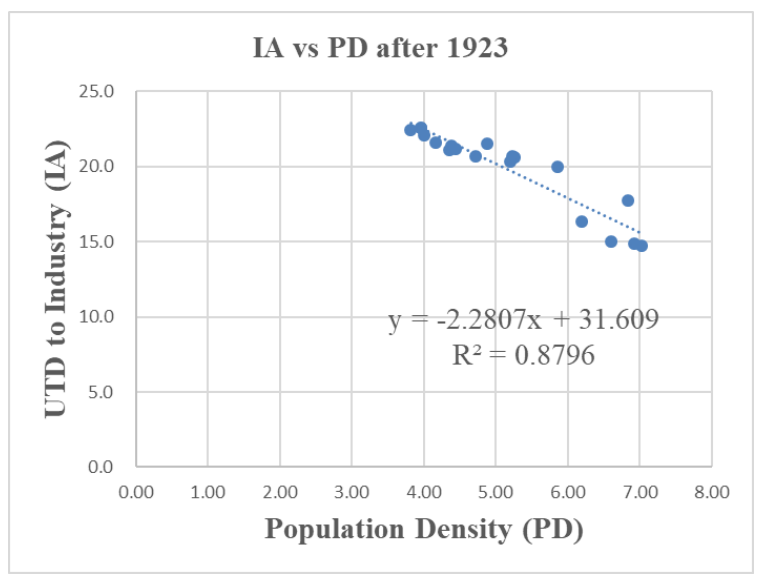

(m)

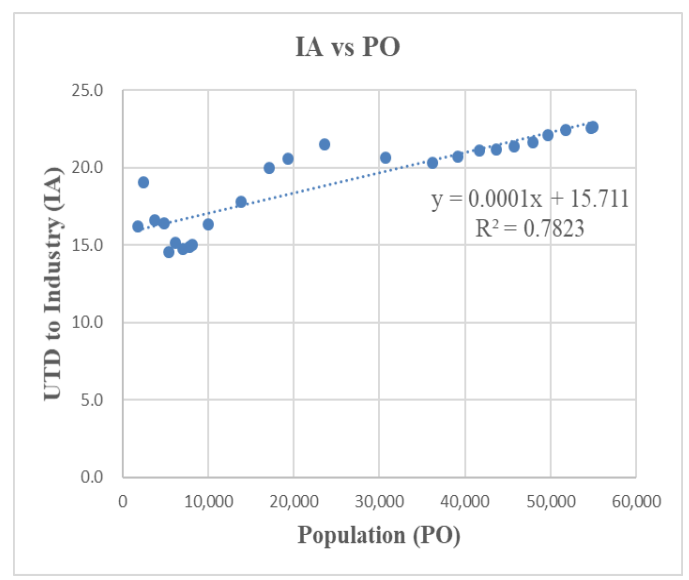

(j)

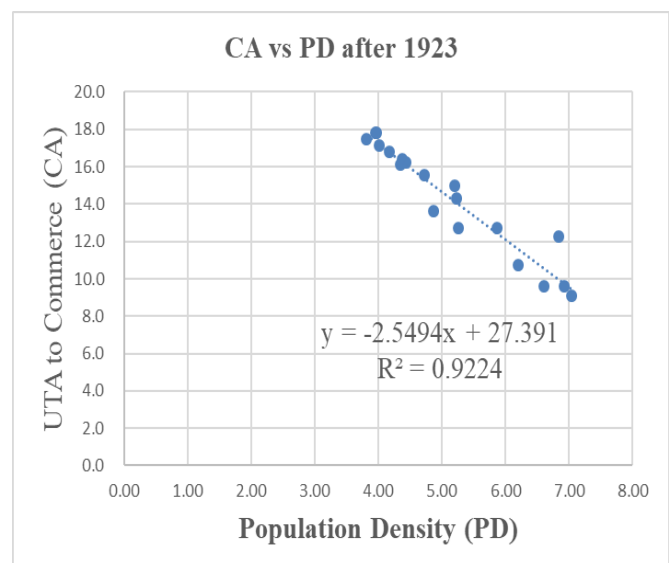

(1)

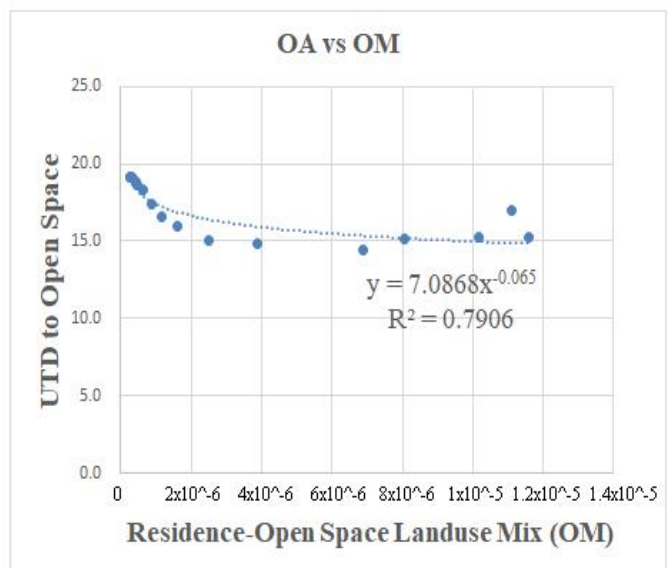

(n)

Figure 8. Cont. 


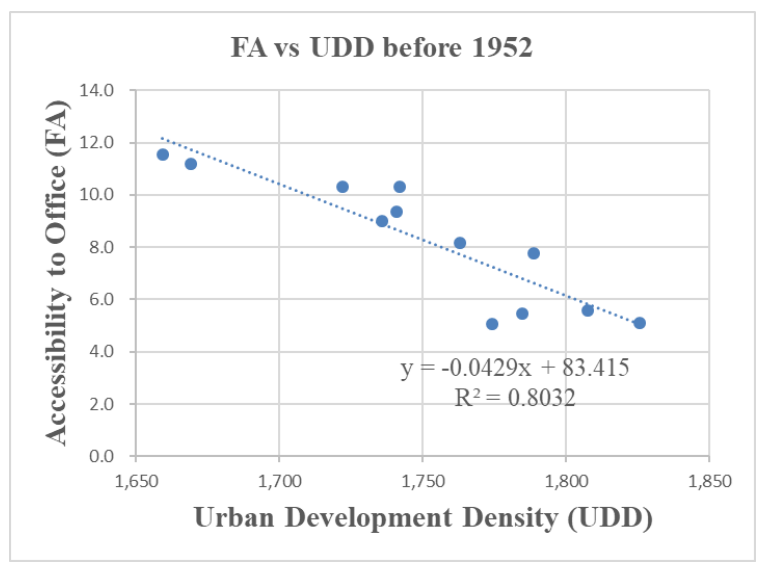

(o)

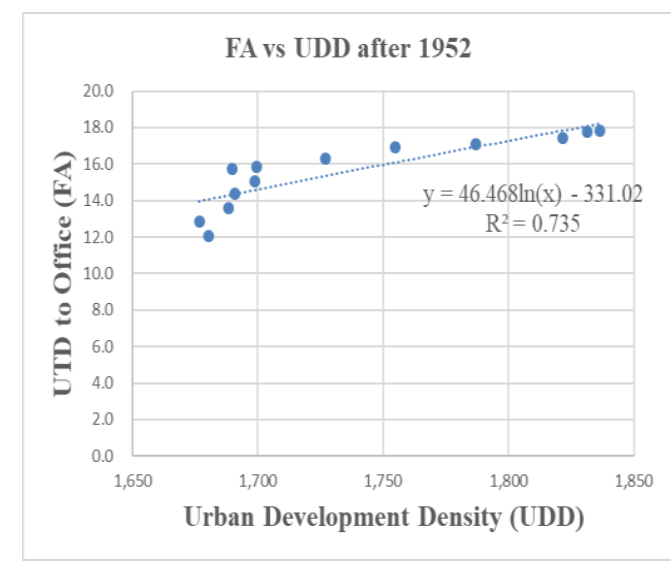

(p)

Figure 8. Sample Strong Linear and Non-Linear Relationships. (a) Correlation between PO and P; (b) Correlation between UD and P; (c) Correlation between CP and RS; (d) Correlation between CA and UD; (e) Correlation between CA and CM; (f) Correlation between FA and FM; (g) Correlation between IA and IM; (h) Correlation between TA and LM; (i) Correlation between CA and PO; (j) Correlation between IA and PO; (k) Correlation between FA and PO; (1) Correlation between CA and PD after 1923; (m) Correlation between IA and PD after 1923; (n) Correlation between OA and OM; (o) Correlation between FA and UDD before 1952; (p) Correlation between FA and UDD after 1952.

Also, the UTD to commercial and industrial parcels is positively linear-correlated with the population (Figure $8 \mathbf{i}-\mathbf{j}$ ), which is positively power-correlated with the average UTD to office (Figure 8k). No clear correlation is found between the population density and the development density throughout the City's history. This confirms the general observation that small to medium-sized cities typically have a flat density curve [63].

Some correlations changed with time. For example in the historical periods after 1923, the average UTD to commerce, industry and the average UTD to office are all negatively linear-correlated with the population density (Figure 81-m), which indicates theoretically that increasing population density is an effective measure to reducing urban travel distances, in practice, however, increasing the population density in the suburban area is difficult due to the cultural and demographic characteristics [64]. The UTD from residential parcels to open space is highly negatively correlated with the land-uses mixed degree from 1908 to 2014 with $R^{2}$ as high as 0.79 (Figure $8 \mathrm{n}$ ). But these correlations are not clear in the City's early historical development periods (before the early of the 20th century) and this could be explained by the fact that during a city's early historical development periods, urbanized areas were typically more scattered around and their spatial interactions were relatively low $[1,65]$.

Prior to 1952, the average UTD to office was negatively linear-correlated with the urbanization density (Figure 8o). However, after 1952, this correlation turned into positive (Figure 8p). This indicated that increasing urban development density and floor area ratio does not necessarily mean to change people's travel mode in this small to medium-sized American city. This might be attributed to the high automobile ownership in the small to medium-sized city and the fact that people prefer to live in low-rise housing estates with more private space on the edge of the city rather than the apartments at the urban core $[66,67]$. Also, the individual property size has been increasing over the past century on the sprawling land parcels, and some urban facilities have been shifting to larger scales but with less quantities [68,69].

\section{Conclusions}

This research applies three quantitative models - growth, urban travel distance, and correlation to provide important anatomic insights into Corvallis, a small-to-medium sized American city, on its historical urban growth patterns and urban form-urban travel distance relationships in space and time. 
The model is based on a detailed citywide tax-lot parcel database containing necessary data on land use, year of built, building square footage, parcel acreage, and property value, which are further linked to socio-economic data to yield urban density, accessibility, and growth, and form.

This study could be improved in several ways. First, urban form, growth, and density measured over building square footage and land use parcel acreage, separately or cumulatively by time period, may not be accurate enough since some buildings are multi-story and some large parcels are only partially urbanized. Second, the average distance from all residential parcels to other land use parcels was used to indicate urban accessibility. However, more realistic accessibility measures based on individual homes to urban facilities may be needed. Such accessibility can also be enhanced by using real urban street networks and transportation modes, and investigating how people's activities influenced their social democratic attributes. Third, due to the minimum number of subject requirements [70], we could not carry out multiple variate analysis in this research. Statistical relationships through simple correlation and linear regression, while not able to reveal causality, may be enhanced by additional qualitative research, such as historical, content, or phenomenological studies, especially for drastic changes in population flows, urban growth, form, and accessibility in space and time. Last but not least, the analysis based on one city in the USA and so is not widely representative, and there is a need to carry similar tests in comparable cities. All of these issues or limitations call for further investigations.

The results in Corvallis indicate that the growth of the total urbanized parcel and the building size had very similar trends. Residential and commercial areas have always been growing with different rates throughout history, and residential land-use consistently accounted for a larger proportion of urbanized lands than any other non-residential land uses. The industrial, office and open space grew rapidly during a few historical periods, but had little growth during other historical periods. Due to the zoning and development policy in the City, the average travel distance from residential to commercial and office were evidently shorter than the average travel distance from residential to industrial through the City's growth periods. The average travel distance from residential land use to office and commercial land uses were growing in a very similar pattern due to the retail-office development and construction [55]. Through the correlation and regression analysis, it is found that different from previous studies on metropolitan areas [19], the urban density increases steadily after the middle part of the 20th century in Corvallis. But the increasing of urban development density and floor area ratio does not necessarily lead to a change in people's travel mode and accessibility in this typical American city, which is possibly due to the popularity of the automobile, the lack of urban facilities in nearby neighborhoods such as groceries, parks and schools [67] and preferences to live in low-rise housing estates with more private space on the edge of the city rather than the apartments at the urban core [68].

From this study we would suggest that there is a primary outcome that might be advisable for urban authorities to consider, subject to further testing and verification in other, similar cities. This is notably the promotion of mixed-use neighborhoods in small to medium-sized cities to alter the commuting pattern; this is based on the finding that increasing the level of land-uses mixture, for example mixed use neighborhoods development [29], is more effective and feasible in reducing urban travel distances; in Corvallis this concurrently improved urban accessibility and was fundamental to achieving sustainable urban growth and form for small-to-medium sized urban cities. Indeed, the findings showed that in Corvallis land-use mix was a more effective and feasible approach in reducing urban travel path distance and enhance accessibility than increasing population density or urban development density. Such a finding has potentially significant implications for our understanding of urban development and planning.

Author Contributions: Z.L. provided the initial concept, research design, data collection, analysis approach and wrote the manuscript. S.G. helped data collection, analysis approach and revision of the manuscript. W.Y., B.R., C.T. and W.C. helped revise the manuscript. B.R. improved the English. All authors read and approved the final manuscript. 
Funding: This research was funded by National Natural Science Foundation of China (No. 51708399); Macao Foundation and Macao Cultural Affairs Bureau Academic Research Grant (No. 2018). And the APC was funded by City University of Macau Foundation.

Conflicts of Interest: The authors declare no conflict of interest.

\section{Appendix A}
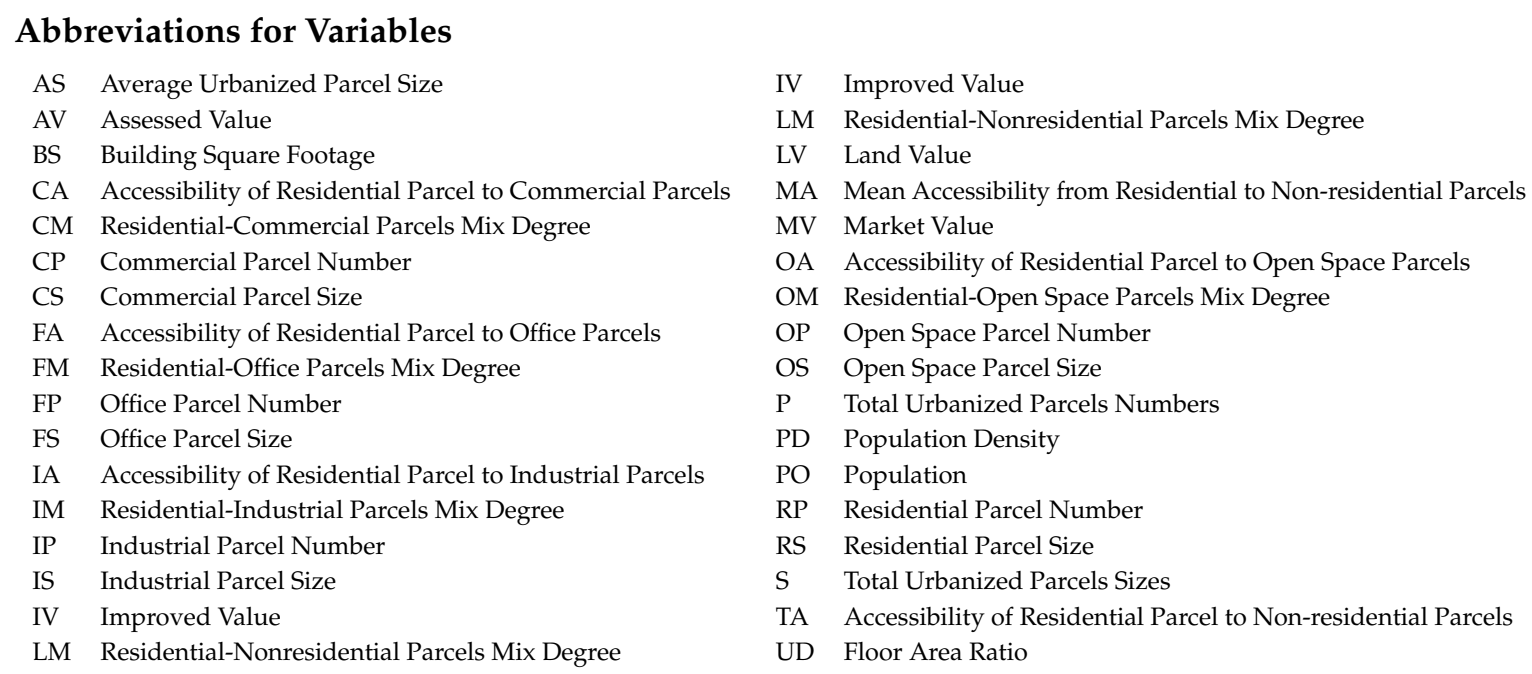

\section{References}

1. Lothrop, J.C.; Lowery, D.L.; Spiess, A.E.; Ellis, C.J. Early Human Settlement of Northeastern North America. PaleoAmerica 2016, 2, 192-251. [CrossRef]

2. Livability. Top 100 Best Places to Live. 2018. Available online: https://livability.com/best-places/top-100best-places-to-live/2018 (accessed on 16 April 2018).

3. Shahraki, S.Z.; Sauri, D.; Serra, P.; Modugno, S.; Seifolddini, F.; Pourahmad, A. Urban sprawl pattern and land-use change detection in Yazd, Iran. Habitat Int. 2011, 35, 521-528. [CrossRef]

4. Center for International Earth Science Information Network (CIESIN). Gridded Population of the World: Land and Water Area. 2010. Available online: https:/ / doi.org/10.7927/H4Z03642 (accessed on 8 June 2018).

5. UN Habitat. Planning and Design for Sustainable Urban Mobility: Global Report on Human Settlements 2013. 2013. Available online: https:// unhabitat.org/planning-and-design-for-sustainable-urban-mobilityglobal-report-on-human-settlements-2013/ (accessed on 6 May 2018).

6. Nam, K.; Lim, U.; Kim, B.H.S. 'Compact' or 'Sprawl' for sustainable urban form? Measuring the effect on travel behavior in Korea. Ann. Reg. Sci. 2012, 49, 157-173. [CrossRef]

7. Zhao, P.J. Sustainable urban expansion and transportation in a growing megacity: Consequences of urban sprawl for mobility on the urban fringe of Beijing. Habitat Int. 2010, 34, 236-243. [CrossRef]

8. Tana Kwan, M.P.; Chai, Y.W. Urban form, car ownership and activity space in inner suburbs: A comparison between Beijing (China) and Chicago (United States). Urban Stud. 2016, 53, 1784-1802. [CrossRef]

9. Chen, Y.J.; Akar, G. Using trip chaining and joint travel as mediating variables to explore the relationships among travel behavior, socio-demographics, and urban form. J. Transp. Land Use 2017, 10, 573-588. [CrossRef]

10. Scheiner, J. Social inequalities in travel behaviour: Trip distances in the context of residential self-selection and lifestyles. J. Transp. Geogr. 2010, 18, 679-690. [CrossRef]

11. Steiner, J.F. Mitaka, from Village to Suburban City: A Study of Tokyo's Urban Fringe: A Condensed English Version; Rural Welfare Research Institute: Tokyo, Japan, 1957; ASIN B0006D0LLO.

12. Sykora, L.; Mulicek, O. Territorial arrangements of small and medium-sized towns from a functional-spatial perspective. Tijdschr. Econ. Soc. Geogr. 2017, 108, 438-455. [CrossRef]

13. Cox, E.; Longlands, S. The Role of Small and Medium-Sized Towns and Cities in Growing the Northern Powerhouse. 2016. Available online: https:/ / www.ippr.org/files/publications/pdf/city-systems_June2016.pdf (accessed on 16 July 2018). 
14. Wang, Y.; Jin, C.; Lu, M.Q.; Lu, Y.Q. Assessing the suitability of regional human settlements environment from a different preferences perspective: A case study of Zhejiang Province, China. Habitat Int. 2017, 70, 1-12. [CrossRef]

15. Jacob, J. The Economy of Cities; Random House: New York, NY, USA, 1970; ISBN 9780525432869.

16. Mumford, L. The natural history of urbanization. In Man's Role in Changing the Face of the Earth; Thomas, E.L., Ed.; University of Chicago Press: Chicago, IL, USA, 1956; pp. 386-397, ISBN 9780758153319.

17. Yue, W.Z.; Zhang, L.L.; Liu, Y. Measuring sprawl in large Chinese cities along the Yangtze River via combined single and multidimensional metrics. Habitat Int. 2016, 57, 43-52. [CrossRef]

18. Lefebvre, H. Le Manifeste Différentialiste [Differentialist Manifesto]; Gallimard: Paris, France, 1970; ISBN 9782072419355.

19. Ewing, R.; Cervero, R. Travel and the built environment. J. Am. Plan. Assoc. 2010, 76, 265-294. [CrossRef]

20. Frank, L.D.; Engelke, P.O. The built environment and human activity patterns: Exploring the impacts of urban form on public health. J. Plan. Lit. 2001, 16, 202-218. [CrossRef]

21. Hao, P.; Geertman, S.; Hooimeijer, P.; Sliuzas, R. The land-use diversity in urban villages in Shenzhen. Environ. Plan. A 2012, 44, 2742-2764. [CrossRef]

22. Wu, J.S.; Ma, L.; Li, W.F.; Peng, J.; Liu, H. Dynamics of urban density in China: Estimations based on DMSP/OLS nighttime light data. J. Sel. Top. Appl. Earth Obs. Remote Sens. 2014, 7, 4226-4275. [CrossRef]

23. Noland, R.B.; Karathodorou, N.; Graham, D. Estimating the effect of urban density on fuel demand. Energy Econ. 2010, 32, 86-92. [CrossRef]

24. Coutts, A.M.; Beringer, J.; Tapper, N. Impact of increasing urban density on local climate: Spatial and temporal variations in the surface energy balance in Melbourne, Australia. J. Appl. Meteorol. Clim. 2007, 46, 477-493. [CrossRef]

25. Titman, S.; Twite, G. Urban density, law and the duration of real estate leases. J. Urban Econ. 2013, 74, 99-112. [CrossRef]

26. Song, Y.; Shao, G.F.; Song, X.D.; Liu, Y.; Pan, L.; Ye, H. The relationships between urban form and urban commuting: An empirical study in China. Sustainability 2017, 9, 17. [CrossRef]

27. Naess, P. New urbanism or metropolitan-level centralization? A comparison of the influences of metropolitan-level and neighborhood-level urban form characteristics on travel behavior. J. Transp. Land Use 2011, 4, 25-44. [CrossRef]

28. Gehrke, S.R.; Clifton, K.J. Operationalizing land use diversity at varying geographic scales and its connection to mode choice evidence from Portland, Oregon. Transp. Res. Rec. 2014, 2453, 128-136. [CrossRef]

29. Bahadure, S.; Kotharkar, R. Assessing sustainability of mixed use neighborhoods through residents' travel behavior and perception: The case of Nagpur, India. Sustainability 2015, 7, 12164-12189. [CrossRef]

30. Lewis, S. Neighborhood density and travel mode: New survey findings for high densities. Int. J. Sustain. Dev. World 2018, 25, 152-165. [CrossRef]

31. Zhou, L.; Shen, G.Q.; Woodfin, T.; Chen, T.; Song, K. Ecological and economic impacts of green roofs and permeable pavements at the city level: The case of Corvallis, Oregon. J. Environ. Plan. Manag. 2017, 61, 430-450. [CrossRef]

32. Frank, L.D.; Pivo, G. Impact of Mixed Use and Density on Three Modes of Travel: Single-Occupant Vehicle, Transit, Walking. Available online: http:/ / citeseerx.ist.psu.edu/viewdoc/download?doi=10.1.1.368.4497\& rep=rep1\&type $=$ pdf (accessed on 22 November 2018).

33. Sanchez, T.W. The connection between public transit and employment-The cases of Portland and Atlanta. J. Am. Plan. Assoc. 1999, 65, 284-296. [CrossRef]

34. Levine, J. Rethinking accessibility and jobs-housing balance. J. Am. Plan. Assoc. 1998, 64, 133-149. [CrossRef]

35. Anderson, W.P.; Kanaroglou, P.S.; Miller, E.J. Urban form, energy and the environment: A review of issues, evidence and policy. Urban Stud. 1996, 33, 7-35. [CrossRef]

36. Bhat, C.; Handy, S.; Kockelman, K.; Mahmassani, H.; Chen, Q.L.; Weston, L. Urban Accessibility Index: Literature Review. 2000. Available online: http://ctr.utexas.edu/wp-content/uploads/pubs/4938_1.pdf (accessed on 16 November 2018).

37. Niedzielski, M.A.; Boschmann, E. Travel Time and Distance as Relative Accessibility in the journey to work. Ann. Assoc. Am. Geogr. 2014, 104, 1156-1182. [CrossRef]

38. Holz-Rau, C.; Scheiner, J.; Sicks, K. Travel distances in daily travel and long-distance travel: What role is played by urban form? Environ. Plan. A 2014, 46, 488-507. [CrossRef] 
39. Boussauw, K.; Witlox, F. Linking expected mobility production to sustainable residential location planning: Some evidence from Flanders. J. Transp. Geogr. 2011, 19, 936-942. [CrossRef]

40. Zhou, L.; Wu, Y.; Woodfin, T.; Zhu, R.; Chen, T. An approach to evaluate comprehensive plan and identify priority lands for future land use development to conserve more ecological values. Sustainability 2018, 10, 126. [CrossRef]

41. Maat, K.; Timmermans, J.P. A causal model relating urban form with daily travel distance through activity/travel decisions. Transp. Plan. Technol. 2009, 32, 115-134. [CrossRef]

42. Pan, H.X.; Shen, Q.; Zhang, M. Influence of urban form on travel behaviour in four neighbourhoods of Shanghai. Urban Stud. 2009, 46, 275-294. [CrossRef]

43. Schlossberg, M.; Greene, J.; Phillips, P.P.; Johnson, B.; Parker, B. School trips-Effects of urban form and distance on travel mode. J. Am. Plan. Assoc. 2006, 72, 337-346. [CrossRef]

44. Grosse, J.; Olafsson, A.S.; Carstensen, T.A.; Fertner, C. Exploring the role of daily "modality styles" and urban structure in holidays and longer weekend trips: Travel behaviour of urban and peri-urban residents in Greater Copenhagen. J. Transp. Geogr. 2018, 69, 138-149. [CrossRef]

45. Corvallis OR. Storm Water Master Plan. 2002. Available online: http://www.corvallisoregon.gov/index. aspx?page=128 (accessed on 2 May 2018).

46. Dempsey, J.A.; Plantinga, A.J. How well do urban growth boundaries contain development? Results for Oregon using a difference-in-difference estimator. Reg. Sci. Urban Econ. 2013, 43, 996-1007. [CrossRef]

47. U.S. Bureau of the Census. State and Country QuickFacts. 2017. Available online: https://www.census.gov / quickfacts/fact/table/US/PST045217 (accessed on 18 April 2018).

48. Corvallis OR. City of Corvallis Land Development Code. 2006. Available online: http://www. corvallisoregon.gov/modules/showdocument.aspx?documentid=8772 (accessed on 18 May 2018).

49. Corvallis OR. GIS Data—Download. 2014. Available online: https://www.corvallisoregon.gov/it/page/ gis-data-download (accessed on 6 May 2018).

50. McKenzie, B. Who Drives to Work? Commuting by Automobile in the United States: 2013. 2015. Available online: https://www.census.gov/content/dam/Census/library/publications/2015/acs/acs32.pdf (accessed on 17 November 2018).

51. ESRI. OD Cost Matrix Analysis. 2018. Available online: http://desktop.arcgis.com/en/arcmap/latest/ extensions/network-analyst/od-cost-matrix.htm (accessed on 17 November 2018).

52. Bayulken, B.; Huisingh, D. A literature review of historical trends and emerging theoretical approaches for developing sustainable cities (part 1). J. Clean. Prod. 2015, 109, 11-24. [CrossRef]

53. Joyanna, M. Recent trends in unemployment and the labor force, 10 countries. 1985. Available online: https: / / www.bls.gov/opub/mlr/1985/08/art2full.pdf (accessed on 20 November 2018).

54. Hodgson, G.M. The great crash of 2008 and the reform of economics. Camb. J. Econ. 2009, 33, $1205-1221$. [CrossRef]

55. Cervero, R. Mixed land-uses and commuting: Evidence from the American housing survey. Transp. Res. A 1996, 30, 361-377. [CrossRef]

56. Szczygiel, B. "City beautiful” revisited-An analysis of nineteenth-century civic improvement efforts. J. Urban Hist. 2003, 29, 107-132. [CrossRef]

57. Martinez-Fernandez, C.; Audirac, I.; Fol, S.; Cunningham-Sabot, E. Shrinking cities: Urban challenges of globalization. Int. J. Urban Reg. 2012, 36, 213-225. [CrossRef]

58. Kim, S. Industrialization and urbanization: Did the steam engine contribute to the growth of cities in the United States? Explor. Econ. Hist. 2005, 42, 586-598. [CrossRef]

59. Brown, J.R.; Morris, E.A.; Taylor, B.D. Planning for cars in cities: Planners, engineers, and freeways in the 20th Century. J. Am. Plan. Assoc. 2009, 75, 161-177. [CrossRef]

60. Wei, Y.P.; Zhang, Z.Y. Assessing the fragmentation of construction land in urban areas: An index method and case study in Shunde, China. Land Use Policy 2012, 29, 417-428. [CrossRef]

61. Veiga, L.; Magrini, A. Eco-industrial park development in Rio de Janeiro, Brazil: A tool for sustainable development. J. Clean. Prod. 2009, 17, 653-661. [CrossRef]

62. Hersperger, A.M.; Franscini, M.P.G.; Kubler, D. Actors, Decisions and Policy Changes in Local Urbanization. Eur. Plan. Stud. 2014, 22, 1301-1319. [CrossRef]

63. Talen, E. Traditional urbanism meets residential affluence-An analysis of the variability of suburban preference. J. Am. Plan. Assoc. 2001, 67, 199-216. [CrossRef] 
64. Chi, G.Q.; Ventura, S. Population change and its driving factors in rural, suburban, and urban areas of Wisconsin, USA, 1970-2000. Int. J. Popul. Res. 2011, 2011, 856534. [CrossRef]

65. Hasan, S.; Wang, X.M.; Khoo, Y.B.; Foliente, G. Accessibility and socio-economic development of human settlements. PLoS ONE 2017, 12, e0179620. [CrossRef] [PubMed]

66. Southworth, M.; Owens, P.M. The evolving metropolis-studies of community, neighborhood, and Street form at the urban edge. J. Am. Plan. Assoc. 1993, 59, 271-287. [CrossRef]

67. U.S. EPA (Environmental Protection Agency). Travel and Environmental Implications of School Siting. 2003. Available online: https://www.epa.gov/sites/production/files/2014-04/documents/school_travel.pdf (accessed on 16 November 2018).

68. Kempen, E.; Musterd, S. High-rise housing reconsidered: Some research and policy-implications. Hous. Stud. 1991, 6, 83-95. [CrossRef]

69. Muresan, A. Who Lives Largest? The Growth of Urban American Homes in the Last 100 Years. Available online: https:/ / www.propertyshark.com/Real-Estate-Reports/2016/09/08/the-growth-of-urbanamerican-homes-in-the-last-100-years/ (accessed on 20 November 2018).

70. Green, S.B. How many subjects does it take to do a regression analysis. Multivar. Behav. Res. 1991, 26, 499-510. [CrossRef] [PubMed]

(C) 2018 by the authors. Licensee MDPI, Basel, Switzerland. This article is an open access article distributed under the terms and conditions of the Creative Commons Attribution (CC BY) license (http://creativecommons.org/licenses/by/4.0/). 